\title{
Towards Gauging Time-Reversal Symmetry: A Tensor Network Approach
}

\author{
Xie Chen ${ }^{1,2}$ and Ashvin Vishwanath ${ }^{2}$ \\ ${ }^{1}$ Department of Physics and Institute for Quantum Information and Matter, \\ California Institute of Technology, Pasadena, California 91125, USA \\ ${ }^{2}$ Department of Physics, University of California, Berkeley, California 94720, USA \\ (Received 20 April 2015; revised manuscript received 4 November 2015; published 30 November 2015)

\begin{abstract}
It is well known that unitary symmetries can be "gauged," i.e., defined to act in a local way, which leads to a corresponding gauge field. Gauging, for example, the charge-conservation symmetry leads to electromagnetic gauge fields. It is an open question whether an analogous process is possible for time reversal which is an antiunitary symmetry. Here, we discuss a route to gauging time-reversal symmetry that applies to gapped quantum ground states that admit a tensor network representation. The tensor network representation of quantum states provides a notion of locality for the wave function coefficient and hence a notion of locality for the action of complex conjugation in antiunitary symmetries. Based on that, we show how time reversal can be applied locally and also describe time-reversal symmetry twists that act as gauge fluxes through nontrivial loops in the system. As with unitary symmetries, gauging time reversal provides useful access to the physical properties of the system. We show how topological invariants of certain timereversal symmetric topological phases in $D=1,2$ are readily extracted using these ideas.
\end{abstract}

\section{INTRODUCTION}

For condensed matter systems with global symmetry, coupling to the corresponding gauge field provides a useful access to the physical properties of the system. For example, in systems with charge-conservation [U(1)] symmetry, coupling to the electromagnetic field and measuring the induced charge or current is a direct probe of the lowenergy excitations of the system. In gapped systems without low-energy excitations, coupling to gauge field and introducing gauge fluxes to the system creates finite-energy excitations that can reveal important information about the topological order of the system. For example, in fractional quantum Hall systems with U(1) symmetry, inserting a magnetic flux $\Phi$ results in the accumulation of charge $\sigma_{x y} \Phi$ around the flux and hence is a direct measure of the quantized Hall conductance [1]. When the continuous U(1) symmetry is broken down to a discrete symmetry, discrete fluxes can be introduced. For example, in superconductors where the $\mathrm{U}(1)$ symmetry breaks down to $Z_{2}$, fluxes in multiples of $h c / 2 e$ can penetrate the system, and one of the most important properties of the topological $p+i p$ superconductor is that each $h c / 2 e$ flux contains a Majorana zero mode [2]. Similarly, systems with non-Abelian symmetries can be coupled to non-Abelian gauge fields [3]. In general, coupling topological phases with various symmetries [symmetry-protected topological (SPT) phases or

Published by the American Physical Society under the terms of the Creative Commons Attribution 3.0 License. Further distribution of this work must maintain attribution to the author(s) and the published article's title, journal citation, and DOI. symmetry-enriched topological (SET) phases] to the corresponding gauge field results in nontrivial responses (like nontrivial statistics, Hall conductance, symmetry fractionalization, etc.) and provides an important tool in distinguishing these phases [4-10].

Can time-reversal symmetry be similarly gauged? The antiunitary nature of time-reversal symmetry has made such a notion hard to define, and we can see how a straightforward generalization from unitary symmetries fails. In coupling systems with global unitary symmetries to gauge fields, we first find the action of the symmetry on the local Hilbert spaces in the system. For U(1) symmetry, it would be adding phase factors on the local charges, and for $\mathrm{SU}(2)$ symmetry, it would be local rotation of spins. Then extra degrees of freedom-the gauge field-are introduced into the system that transform under the local symmetry and couple to the original degrees of freedom in such a way that the total system is now invariant under arbitrary local actions of the symmetry. However, when trying to implement the same procedure for time-reversal symmetry, we fail at the first step-we do not know how to define a local action of time reversal. Global time-reversal symmetry involves not only unitary transformations on local Hilbert spaces (like inverting spins) but also a complex conjugation operation on the coefficient of each basis state in the wave function:

$$
\begin{aligned}
\mathcal{T} & \sum_{i_{1}, \ldots, i_{N}} C_{i_{1}, \ldots, i_{N}}\left|i_{1}, \ldots, i_{N}\right\rangle \\
& =\sum_{i_{1}, \ldots, i_{N}} C_{i_{1}, \ldots, i_{N}}^{*} U_{1} \otimes \cdots \otimes U_{N}\left|i_{1}, \ldots, i_{N}\right\rangle .
\end{aligned}
$$


Therefore, to couple a system to time-reversal gauge field, first we need to define how complex conjugation acts on $C_{i_{1}, \ldots, i_{N}}$ locally. (The local action of the unitary part is straightforwardly defined.)

To define such an action, we need to decide if we should consider $C_{i_{1}, \ldots, i_{N}}$ to be localized on a particular lattice site or distributed over the entire system. Different ways to divide the coefficient would result in different local actions of time reversal. If we designate $C_{i_{1}, \ldots, i_{N}}$ to be localized on site 1 , then local action of time reversal on site 1 takes complex conjugation of $C_{i_{1}, \ldots, i_{N}}$ while local actions on other sites do not. If we think of $C_{i_{1}, \ldots, i_{N}}$ as composed of various parts on different lattice sites, then time reversal on a subsystem takes complex conjugation of the corresponding part and not the others. Then the question is, what is the most meaningful way to do this?

Useful insight can be obtained by thinking of a simple case-a product state:

$$
|\psi\rangle=\prod_{k}\left(a^{0_{k}}|0\rangle+a^{1_{k}}|1\rangle\right)=\sum_{i_{1}, \ldots, i_{N}} a^{i_{1}} \ldots a^{i_{N}}\left|i_{1}, \ldots, i_{N}\right\rangle .
$$

In such a product state, it is natural to divide $C_{i_{1}, \ldots, i_{N}}$ into $N$ parts $a^{i_{k}}, k=1, \ldots, N$, and associate them with each site $k$. Acting time reversal locally on site $k$ then involves taking complex conjugation on $a^{i_{k}}$. With this definition of local time-reversal symmetry action, we can see that if the total state $|\psi\rangle$ is invariant under global time-reversal action, then it is also invariant under local time-reversal action (up to a phase factor). This is similar to the action of unitary symmetries on a product symmetric state.

How then do we divide the wave function coefficient $C_{i_{1}, \ldots, i_{N}}$ in a many-body entangled state? The tensor network representation of many-body entangled states [11,12] provides a very natural way to do so. The tensor network representation describes a many-body wave function in terms of a set of local tensors $T^{i_{k}}$,

$$
|\psi\rangle=\sum_{i_{1}, \ldots, i_{N}} t \operatorname{Tr}\left(T^{i_{1}} \ldots T^{i_{N}}\right)\left|i_{1}, \ldots, i_{N}\right\rangle
$$

where $t \operatorname{Tr}$ denotes tensor contraction. We can then think of the tensors $T^{i_{k}}$ as local pieces of the total coefficient $C_{i_{1}, \ldots, i_{N}}$ and define local action of complex conjugation on site $k$ as taking complex conjugation of $T^{i_{k}}$. Combined with the local action of the unitary part of time reversal, we obtain a local way of implementing time-reversal symmetry. In this paper, we focus on gapped short-range correlated quantum states that can be well described using the tensor network formalism, and such a definition of local time-reversal action applies [13].

Is this a valid and useful definition? First, we notice that such a local action of time reversal leads to similar changes

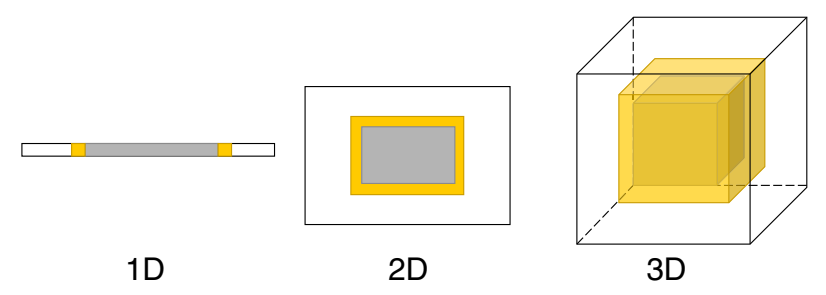

FIG. 1. Local action of time-reversal symmetry (gray and yellow region) on a short-range correlated symmetric state changes only the state at the border of the region (yellow region).

on a gapped symmetric quantum state as that induced by the local action of unitary symmetries. Imagine applying a unitary symmetry to a subregion in a gapped symmetric quantum state. Both deep inside and outside the region, the state should remain invariant. The only change in the state happens at the border of the region of symmetry action, as shown in Fig. 1. To see how this can be true in our definition of local time-reversal symmetry action on tensor network states, we note that global time-reversal symmetry involves complex conjugation on all the tensors $T^{i_{k}}$ and unitary operations $\prod_{k} U_{k}$ on all the physical degrees of freedom. The tensors at site $k\left(T^{i_{k}}\right)$ may change into $\tilde{T}^{i_{k}}$ under complex conjugation and $U_{k}$. However, if the state is invariant under global time-reversal symmetry action, then the changes in $T^{i_{k}}$ should cancel with that coming from neighboring sites. Because of this, if we apply time-reversal symmetry locally (complex conjugation and $U_{k}$ on sites in a subregion), then tensors both inside and outside the subregion remain effectively invariant while tensors along the border can change. Therefore, intuitively, this definition of local time-reversal symmetry action changes the quantum state in a way we would expect.

More concretely, we show in the following sections that by using such a definition of local time-reversal symmetry action we can extract topological invariants from a gapped symmetric quantum states and hence identify the symmetry-related topological order [15]. Unlike unitary symmetries though, the time-reversal symmetry twists we introduce are always confined. Whether they can be made deconfined by introducing dynamic time-reversal gauge field is an open issue, which we discuss at the end of this paper.

In Sec. II, we discuss the 1D case in terms of matrix product states (MPS) and present a way to insert timereversal fluxes through a $1 D$ ring. In $1 D$ there are two different time-reversal symmetry-protected topological phases [16-18]. We demonstrate how these two phases can be distinguished from each other using the projective composition rule of time-reversal twists induced by the inserted time-reversal fluxes. That is, two time-reversal twists compose into identity up to a universal phase factor characterizing the underlying SPT order of the state. In 1D, the distinction between different time-reversal SPT phases has been well understood in the matrix product formalism 
[17-20]. Our discussion is just a reinterpretation of that procedure in terms of local time-reversal symmetry action and time-reversal twists.

In Sec. III, we move on to the 2D case, where we define local action of time reversal in tensor product states and discuss how to insert time-reversal fluxes through nontrivial loops in the systems. To facilitate discussion, we first review the procedure for the unitary $Z_{2}$ symmetry and demonstrate how topological invariants of the $2 \mathrm{D} Z_{2}$ SPT phases can be extracted from the projective composition rules of $Z_{2}$ symmetry twist lines. Then, we study a 2D state with trivial time-reversal SPT order, a 2D SPT phase with $Z_{2} \times Z_{2}^{T}$ symmetry, and a $2 \mathrm{D} Z_{2}$ gauge theory with timereversal symmetry and see how the topological invariants of these phases can be extracted similarly. In particular, we find that for the trivial time-reversal SPT state the projective composition rule for the time-reversal fluxes are all trivial, as expected. For the $Z_{2} \times Z_{2}^{T}$ SPT state and the $Z_{2}$ gauge theory with time-reversal symmetry, a nontrivial $(-1)$ phase factor can appear which is related to the $\mathcal{T}^{2}=-1$ transformation law of the $Z_{2}$ fluxes in the bulk. In Sec. IV, we summarize what we learn and discuss open problems in gauging time-reversal symmetry.

Throughout this paper, $Z_{2}$ refers to either a unitary $Z_{2}$ symmetry or the corresponding $Z_{2}$ gauge symmetry (and related topological order). $Z_{2}^{T}$ is used for time-reversal symmetry. $\mathbb{Z}_{2}$ is used to denote classification of phases.

\section{1D MATRIX PRODUCT STATES}

The matrix product state representation of 1D gapped quantum states provides a natural way to divide the wave function coefficient into local pieces. The matrix product state representation reads

$$
|\psi\rangle=\sum_{i_{1}, i_{2}, \ldots, i_{N}} \operatorname{Tr}\left(A^{i_{1}} A^{i_{2}} \ldots A^{i_{N}}\right)\left|i_{1}, i_{2}, \ldots, i_{N}\right\rangle,
$$

with $D \times D$ matrices $A^{i}$. We call the $i$ 's the physical indices and the left and right indices of $A$ 's the inner indices. Similar terminology is used for tensor product states discussed later. The local action of time-reversal symmetry on matrix product states has been discussed extensively in the study of 1D symmetry-protected topological phases [17-20]. Here, we review the procedure and discuss the notion of time-reversal flux and time-reversal twists based on such a formalism.

Suppose that the global time-reversal symmetry action is $U \otimes \cdots \otimes U K$, where $K$ denotes complex conjugation in the $|i\rangle$ basis. The global action of time reversal on a MPS reads

$\mathcal{T}|\psi\rangle=\sum_{i_{1}, i_{2}, \ldots, i_{N}} \operatorname{Tr}\left(A^{i_{1}} A^{i_{2}} \ldots A^{i_{N}}\right)^{*} U \otimes U \cdots \otimes U\left|i_{1} i_{2} \ldots i_{N}\right\rangle ;$ then acting time reversal locally on a single site in the matrix product state changes the matrices to

$$
\tilde{A}^{i}=\sum_{j} U_{i j}^{T}\left(A^{j}\right)^{*}
$$

Note that if this is applied to all sites, then it is equivalent to applying time reversal globally. If the state $|\psi\rangle$ is shortrange correlated and time-reversal symmetric, then the MPS representation satisfies

$$
\tilde{A}^{i}=\sum_{j} U_{i j}^{T}\left(A^{j}\right)^{*}=M A^{i} M^{-1}
$$

with an invertible matrix $M$. Therefore, applying time reversal on one site is equivalent to inserting $M$ and $M^{-1}$ on the two sides of $A^{i}$.

If we apply time reversal to a segment of sites $m$ to $m+n$, then the matrices on all sites remain invariant except for those on site $m$ and $m+n$. On site $m$, the matrices change to

$$
\tilde{A}^{i}=M A_{i}
$$

On site $m+n$, the matrices change into

$$
\tilde{A}^{i}=A^{i} M^{-1} .
$$

For example, consider the dimer state with two spin$1 / 2$ 's per site $\left(\sigma^{l}\right.$ and $\left.\sigma^{r}\right)$ and the spin-1/2's on neighboring sites pair into the singlet state $|01\rangle-|10\rangle$. The Hamiltonian for the state reads

$$
H=\sum_{i} \vec{\sigma}_{i}^{r} \cdot \vec{\sigma}_{i+1}^{l}
$$

The MPS representation of the state contains matrices

$$
\begin{array}{ll}
A^{00}=-|1\rangle\langle 0|, & A^{01}=-|1\rangle\langle 1|, \\
A^{10}=|0\rangle\langle 0|, & A^{11}=|0\rangle\langle 1| .
\end{array}
$$

If time-reversal symmetry acts as

$$
\mathcal{T}=i \sigma_{y} \otimes \cdots \otimes i \sigma_{y} K
$$

then acting time reversal on each site changes the matrices as

$$
\tilde{A}^{i}=(i Y) A^{i}(-i Y)
$$

Here, we use $X, Y$, and $Z$ to denote Pauli matrices on the inner indices.

It is known that the time-reversal SPT order can be extracted from $M$ by [17-20] 


$$
M^{*} M=\beta= \pm 1
$$

where $\beta=1$ in the trivial SPT phase and $\beta=-1$ in the nontrivial one (including the dimer state where $M=i Y$ ). Now we are going to reinterpret this in terms of timereversal fluxes and time-reversal twists.

With the definition of local time-reversal symmetry action, we can discuss how to insert time-reversal fluxes through a $1 \mathrm{D}$ ring, in analogy to unitary symmetries. Let us first recall how the procedure works for unitary symmetries.

Consider, for example, a system with U(1) symmetry $e^{i \theta n_{1}} \otimes e^{i \theta n_{2}} \otimes \cdots \otimes e^{i \theta n_{N}}$, where $n_{k}$ counts the number of $U(1)$ charge on each site. Without loss of generality (WLOG), consider a Hamiltonian with two-body interactions $H=\sum_{k} h_{k, k+1}$. Inserting a $\phi$ flux through the onedimensional ring corresponds to changing the Hamiltonian term on the boundary as

$$
h_{N, 1} \rightarrow e^{i \phi n_{N}} h_{N, 1} e^{-i \phi n_{N}} .
$$

For all the SPT and SET phases we are considering in this paper, the ground states satisfy that all the local reduced density matrices are determined by local Hamiltonian terms and not affected by terms far away (this is the TQO-2 condition used in the definition of topological order in Ref. [21]). Therefore, the two ground states $|\psi\rangle$ and $|\psi\rangle_{\phi}$ without and with flux should have the following relation:

(1) Away from the boundary, $|\psi\rangle$ and $|\psi\rangle_{\phi}$ should look the same.

(2) Near the boundary, $|\psi\rangle_{\phi}$ should look the same as $|\psi\rangle$ with symmetry applied on one side of the boundary, i.e., $e^{i \phi n_{m}} \otimes \cdots \otimes e^{i \phi n_{N}}|\psi\rangle$ or $e^{-i \phi n_{1}} \otimes \cdots \otimes e^{-i \phi n_{m}}|\psi\rangle$, with $1 \ll m \ll N$.

Here, by "look the same" we mean that the two states have the same reduced density matrix locally.

For time-reversal symmetry, we do not know how to couple the system to fluxes on the Hamiltonian level. However, we can couple the symmetric gapped ground state to time-reversal fluxes in a way similar to unitary symmetries. Denote the state not coupled and coupled to a time-reversal flux as $|\psi\rangle$ and $|\psi\rangle_{\mathcal{T}}$, respectively. We expect that

(1) Away from the boundary, $|\psi\rangle$ and $|\psi\rangle_{\mathcal{T}}$ should look the same.

(2) Near the boundary, $|\psi\rangle_{\mathcal{T}}$ should look the same as $|\psi\rangle$ with time reversal applied on one side of the boundary, i.e., from site $m$ to $N$ or from site 1 to $m$, with $1 \ll m \ll N$.

As we discuss above, in short-range correlated matrix product states, local time-reversal symmetry action changes the representing matrices only near the two ends of the local region, as shown in Figs. 2(a) and 2(b). Therefore, if we change the matrices at only one point, as shown in Fig. 2(c), for example, at the boundary of the 1D ring, this would correspond to inserting a time-reversal flux through the $1 \mathrm{D}$ ring.

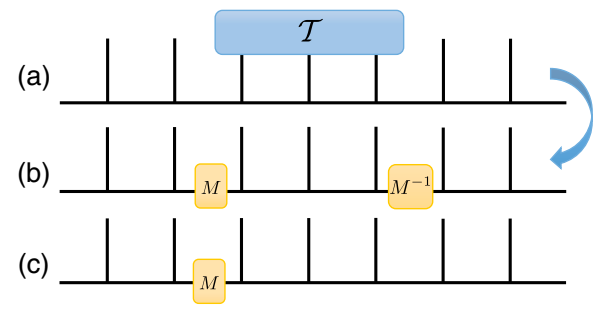

FIG. 2. (a) Local time-reversal symmetry action on a symmetric MPS (b) changes only matrices near the two ends. (c) Coupling a MPS to a time-reversal flux corresponds to changing the matrices at only one place. Vertical links represent physical indices of the MPS and horizontal links represent the inner indices of the MPS.

In particular, to insert a time-reversal flux, we can change the matrices at site $N$ to

$$
A^{i} \rightarrow A^{i} M^{-1}
$$

or we can change the matrices at site 1 to

$$
A^{i} \rightarrow M A^{i}
$$

The resulting state $|\psi\rangle_{\mathcal{T}}$ indeed has the property discussed above when compared to the original state $|\psi\rangle$ without flux and contains a time-reversal symmetry twist on the boundary.

The usefulness of this definition becomes evident when we compose two time-reversal symmetry twists and extract universal properties of the SPT order from the procedure. Suppose that we insert two time-reversal fluxes by changing the matrices on site 1 twice. This should be equivalent to a state without time-reversal flux. However, as we see, two time-reversal twists may differ from zero twist by an important phase factor. On inserting the first flux, the matrices on site 1 are changed to $A^{i} \rightarrow M A^{i}$. On inserting the second flux, the $A_{i}$ part undergoes the change $A^{i} \rightarrow$ $M A^{i}$ again. Moreover, because we are considering timereversal fluxes, we need to take complex conjugation of the first $M$. Therefore, the total change to $A^{i}$ on site 1 is

$$
A^{i} \rightarrow M^{*} M A^{i}=\beta A^{i},
$$

with $\beta=M^{*} M= \pm 1$. Therefore, the composition of two time-reversal twists is equivalent to zero twist up to a phase factor of $\beta$. From this projective composition rule of timereversal twists, we recover the topological invariant $\beta$ characterizing the SPT order of the state. The result remains the same if we insert flux by changing the matrices on site $N$ as $A^{i} \rightarrow A^{i} M^{-1}$.

This corresponds exactly to the procedure of extracting SPT order from the MPS representation of a gapped symmetric state [17-20]. Here, we are merely reinterpreting the procedure as finding the projective composition rule of time-reversal twists induced by inserted timereversal fluxes. It follows from previous discussions that 
the topological invariant extracted $(\beta)$ is independent of the gauge choice we make for the matrix product representation. In particular, we can change the gauge choice of the MPS representation by an invertible matrix $N$. Then, inserting a single flux corresponds to changing the matrices on site 1 by $N^{*} M N^{-1}$ and

$$
\left(N^{*} M N^{-1}\right)^{*}\left(N^{*} M N^{-1}\right)=M^{*} M=\beta .
$$

We note that this procedure applies to any matrix product state, not only the fixed-point ones with zero correlation length as shown in the dimer state example above.

\section{2D TENSOR PRODUCT STATES}

Now, we are ready to generalize the procedure to $2 \mathrm{D}$. First, we review in Sec. III A how everything works for unitary symmetries. In particular, we review how the $Z_{2}$ symmetry acts locally on the tensor product representation of a gapped $Z_{2}$ symmetric state and how topological invariants of its SPT order can be extracted from the projective composition rules of the $Z_{2}$ symmetry twist lines. After this preparation, we move on to define how time-reversal symmetry acts locally on $2 \mathrm{D}$ tensor product states and discuss how to insert time-reversal fluxes through nontrivial loops in the system in Sec. III B. In particular, we discuss in detail three examples in Secs. III B-III D and demonstrate how topological invariants of the phases can be extracted from the projective composition rules of time-reversal twist lines.

\section{A. Review: Local action and twists of unitary symmetry in 2D}

Consider the 2D states with trivial and nontrivial $Z_{2}$ symmetry protected topological order $[4,5,22-24]$. We use a form of wave function similar to that in Ref. [4]. The system lives on a bipartite honeycomb lattice, where each lattice site contains three spin-1/2's (in state $|0\rangle$ or $|1\rangle$ ), as shown in Fig. 3 with three circles. $A$ and $B$ label the two sublattices. In the ground state, the six spin-1/2's around a plaquette are either all in the $|0\rangle$ state or all in the $|1\rangle$ state,

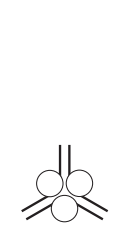

(a)

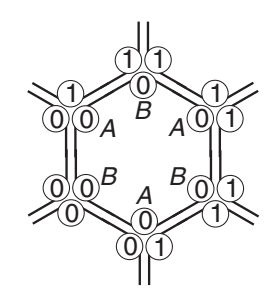

(b)
FIG. 3. Wave function of $2 \mathrm{D}$ states on the honeycomb lattice with $Z_{2}$ SPT order: each lattice site (either $A$ site or $B$ site) contains three spin-1/2's; the six spin-1/2's around each plaquette are either all in the $|0\rangle$ state or all in the $|1\rangle$ state. The $Z_{2}$ symmetry flips between the $|0\rangle$ and $|1\rangle$ state. forming $Z_{2}$ domains. A state with trivial $Z_{2}$ SPT order can be obtained as an equal weight superposition of all $Z_{2}$ domain configurations,

$$
\left|\psi_{0}\right\rangle=\sum_{\mathcal{C}}|\mathcal{C}\rangle
$$

where $\mathcal{C}$ denotes $Z_{2}$ domain configurations. The Hamiltonian of $\left|\psi_{0}\right\rangle$ is simply

$$
H_{0}=-\sum_{p} \tilde{\sigma}_{x}^{p}
$$

where $\tilde{\sigma}_{x}$ flips between the two $Z_{2}$ domains on a plaquette $p:|\tilde{0}\rangle=|000000\rangle,|\tilde{1}\rangle=|111111\rangle$.

A state with nontrivial $Z_{2}$ SPT order takes the form

$$
\left|\psi_{1}\right\rangle=\sum_{\mathcal{C}}(-)^{N_{\mathcal{C}}}|\mathcal{C}\rangle
$$

where $N_{\mathcal{C}}$ counts the number of domain wall loops in the configuration. The Hamiltonian with $\left|\psi_{1}\right\rangle$ as its ground state was given in Ref. [4],

$$
H_{1}=\sum_{p} \tilde{\sigma}_{x}^{p} \prod_{\left\langle p q q^{\prime}\right\rangle} i^{\left(1-\tilde{\sigma}_{z}^{q} \tilde{\sigma}_{z}^{q^{\prime}}\right) / 2},
$$

where $\tilde{\sigma}$ acts on the $Z_{2}$ domains and the product runs over the six vertices around plaquette $p$.

Obviously, these two wave functions are both symmetric under the $Z_{2}$ symmetry action of flipping $Z_{2}$ domains $|0\rangle \leftrightarrow|1\rangle$. However, they contain different SPT orders, which is reflected in the different statistics of their $Z_{2}$ fluxes once the symmetry is gauged. The $Z_{2}$ fluxes have bosonic statistics in the trivial phase represented by $\left|\psi_{0}\right\rangle$ and semionic statistics in the nontrivial phase represented by $\left|\psi_{1}\right\rangle$ [4]. We will see how this difference in SPT order is reflected in the projective composition rule of the $Z_{2}$ symmetry twist lines as discussed below.

It is particularly helpful to use the tensor network representation of the states and see how the tensors transform under the symmetry. For unitary symmetries, the discussion can be carried out without referring to the tensor network representation. But here we use such a representation because (1) it provides a nice picture of how a state changes under local action of symmetry and with the insertion of symmetry fluxes and (2) it paves the way for our discussion of time-reversal symmetry, which necessarily depends on a tensor network representation.

The trivial SPT state $\left|\psi_{0}\right\rangle$ can be represented with tensors given in Fig. 4(a), while the nontrivial SPT state $\left|\psi_{1}\right\rangle$ can be represented with tensors given in Fig. 4(b). Note that a physical index and the two inner indices connected to it are always in the same state $(|0\rangle$ or $|1\rangle)$. Therefore, when the tensors are contracted together, all physical spins around 


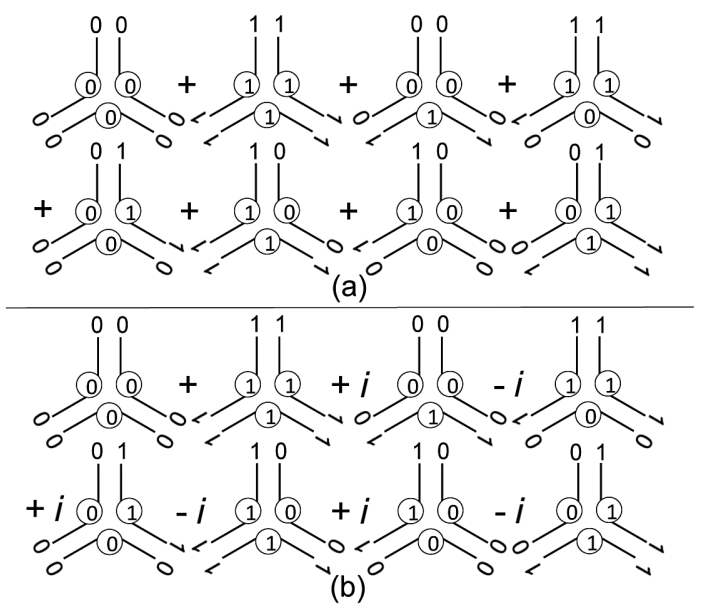

FIG. 4. Tensors representing the trivial $Z_{2}$ SPT wave function in Eq. (20) and the nontrivial $Z_{2}$ SPT wave function in Eq. (22). The labels in circles are physical indices and the labels at the end of the links are inner indices.

the same plaquette are in the same state as shown in Fig. 3, forming $Z_{2}$ domains. The tensor for $\left|\psi_{1}\right\rangle$ is similar to the tensor product representation of the double semion state given in Ref. [25]. The tensors are the same on $A, B$ sublattices. We can see that the tensor network representation for $\left|\psi_{0}\right\rangle$ can be obtained from that for $\left|\psi_{1}\right\rangle$ simply by removing the phase factors $i$ and $-i$ [26].

It is interesting to see how the $Z_{2}$ symmetry acts locally on the tensors. Obviously, the tensors for $\left|\psi_{0}\right\rangle$ and $\left|\psi_{1}\right\rangle$ are not invariant under local action of $Z_{2}$ symmetry $|0\rangle \leftrightarrow|1\rangle$, but the transformed tensors differ from the original ones by unitary transformations on the inner indices. This relation is shown in Fig. 5, where for $\left|\psi_{0}\right\rangle$

$$
\alpha=\bar{\alpha}=1,
$$

and for $\left|\psi_{1}\right\rangle$

$\alpha=|00\rangle\langle 00|+i| 01\rangle\langle 01|+i| 10\rangle\langle 10|+| 11\rangle\langle 11|$,

$\bar{\alpha}=|00\rangle\langle 00|-i| 01\rangle\langle 01|-i| 10\rangle\langle 10|+| 11\rangle\langle 11|$.

$\sigma_{x}$ denotes the spin flip of physical degrees of freedom while $X$ denotes the same operator but on the inner indices. In the following discussion, we call $X \otimes X \alpha$ and $X \otimes X \bar{\alpha}$ the "inner symmetry operators." Using the relation given in

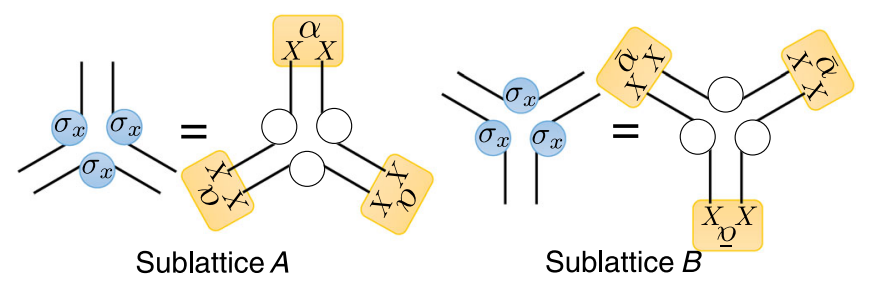

FIG. 5. Local $Z_{2}$ symmetry transformation on the tensors representing the state in Eqs. (20) and (22).
Fig. 5, it is straightforward to see that the state is invariant under global $Z_{2}$ symmetry action and changes only along the border if the $Z_{2}$ symmetry is applied to a subregion. This is because changes to the inner indices cancel if the index lies within the subregion.

Now, we can couple the state to $Z_{2}$ symmetry fluxes. Consider a $Z_{2}$ SPT state on a torus. Inserting a flux through a nontrivial loop of the torus results in a symmetry twist line on the torus along the other nontrivial loop. Assume WLOG that the Hamiltonian of the system contains only two-body interactions. Creating symmetry twist lines corresponds to taking all terms in the Hamiltonian $h_{m n}$ that are divided by the twist line and changing them to

$$
h_{m n} \rightarrow \sigma_{x}^{m} h_{m n} \sigma_{x}^{m} .
$$

We denote the ground state without and with the twist line as $|\psi\rangle$ and $|\psi\rangle_{Z_{2}}$, respectively. Because for the systems under consideration here local reduced density matrices of the ground states are all determined by local Hamiltonian terms, we expect that

(1) Away from the twist line, $|\psi\rangle$ and $|\psi\rangle_{Z_{2}}$ should look the same.

(2) Near the twist line, $|\psi\rangle_{Z_{2}}$ should look like $\prod_{r \in R} \sigma_{x}^{r}|\psi\rangle$, where $R$ is a large region with the twist line as part of the border.

In this way, we can discuss symmetry fluxes in terms of the ground state, instead of the Hamiltonian.

For simplicity of discussion, we combine every two sites on the $A, B$ sublattices and map the system to square lattice structure $\hat{A}_{A}^{B} \longrightarrow-1$. Each tensor now has four inner indices. The inner symmetry operators are $X \otimes X \alpha$ for the up and left indices (inherited from sublattice $A$ ) and $X \otimes X \bar{\alpha}$ for the down and right indices (inherited from sublattice $B$ ).

The tensor product representation of the ground state provides a particularly simple way to find $|\psi\rangle_{Z_{2}}$. As applying symmetry in a region changes the tensors on the border by the inner symmetry operators, as shown in Figs. 6(a) and 6(b), the tensor product representation of $|\psi\rangle_{Z_{2}}$ can be obtained from that of $|\psi\rangle$ by inserting the inner symmetry operators along the twist line, as shown in Fig. 6(c).

In a square lattice tensor product states (TPS) with periodic boundary condition as shown in Fig. 7, threading a $Z_{2}$ flux in the $x$ direction (through the nontrivial loop in the $y$ direction) corresponds to inserting inner symmetry operators, $X \otimes X \alpha$ or $X \otimes X \bar{\alpha}$, along the nontrivial loop in the $x$ direction. Threading a $Z_{2}$ flux in the $y$ direction (through the nontrivial loop in the $x$ direction) corresponds to inserting inner symmetry operators, $X \otimes X \alpha$ or $X \otimes X \bar{\alpha}$, along the twist line of the nontrivial loop in the $y$ direction. Composing two twist lines in the same direction should be equivalent to having no flux in this direction. However, as 


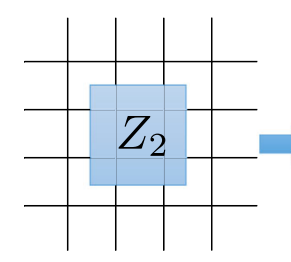

(a)

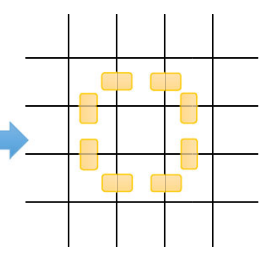

(b)

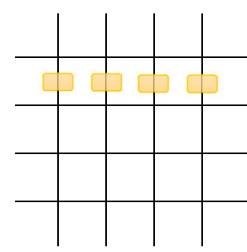

(c)
FIG. 6. (a) Local $Z_{2}$ symmetry action on a symmetric TPS (b) changes only tensors near the border by the inner symmetry operators. (c) Coupling a TPS to a $Z_{2}$ symmetry flux corresponds to changing the tensors by inserting the inner symmetry operators along the twist line. For clarity, physical indices are omitted in this figure. All links represent inner indices of the TPS.

we see, this equivalence is true only up to a phase factor, which is a topological invariant characterizing the underlying SPT order. A similar procedure of inserting symmetry or gauge twist lines and applying modular transformations to extract topological invariants from the states is discussed in Refs. [27,28].

Consider first a state with $Z_{2}$ twist lines only in the $x$ direction. Composing two twist lines, we find that the inner symmetry operator on each inner index composes into

$$
\begin{aligned}
(X \otimes X \alpha)(X \otimes X \alpha) & =I \quad \text { for trivial } \alpha \\
& =Z \otimes Z \text { for nontrivial } \alpha .
\end{aligned}
$$

With trivial $\alpha$, it is obvious that two $Z_{2}$ twist lines compose into zero. With nontrivial $\alpha$, which is illustrated in Fig. 7, the inner symmetry operators on each inner index do not compose into $I$. However, acting $Z \otimes Z$ on all inner indices along a loop (around the boundary of a region) does not change the state at all. This is a special inner symmetry of the tensor product representation given in Fig. 4. Therefore, for both the trivial and nontrivial $Z_{2}$ SPT states, we see that composing two twist lines in the $x$ direction is equivalent to having no twist line, with no extra phase factor.

Nontrivial phase factors can arise when we compose two twist lines in the $x$ direction in the presence of a twist line in the $y$ direction. For the trivial SPT state, the phase factor is

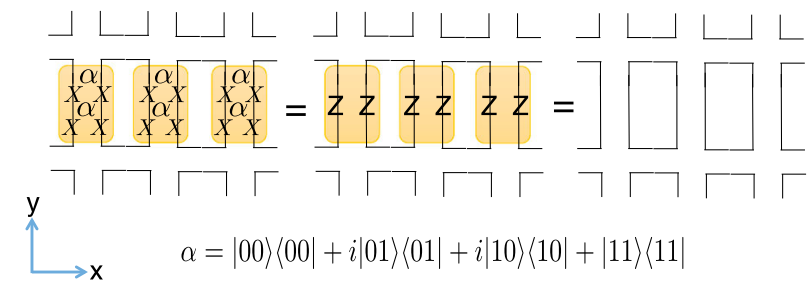

FIG. 7. In the nontrivial $Z_{2}$ SPT state, composing two $Z_{2}$ twist lines in the $x$ direction is equivalent to having no twist lines in the state. The tensor product representation of the state follows from that given in Fig. 4. Physical indices are omitted in the drawing for clarity.

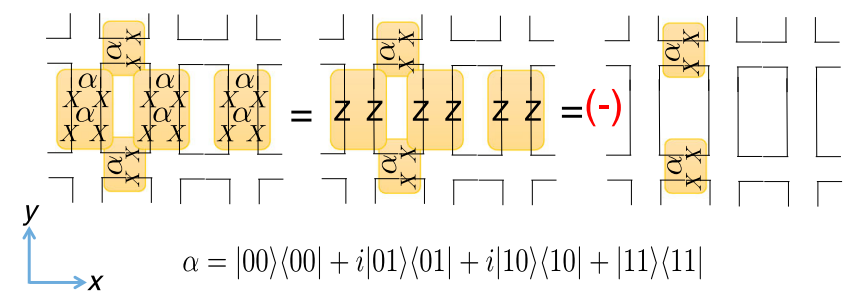

FIG. 8. In the nontrivial $Z_{2}$ SPT state, composing two $Z_{2}$ twist lines in the $x$ direction in the presence of a $Z_{2}$ twist line in the $y$ direction is equivalent to a state with only a $y$ direction twist line up to a -1 phase factor. The tensor product representation of the state follows from that given in Fig. 4. Physical indices are omitted in the drawing for clarity.

still 1 and does not change due to the $y$ direction twist line. However, for the nontrivial SPT state, we can see from Fig. 8 that a -1 phase factor arises. The inner symmetry operators along the $x$ direction compose into $Z \otimes Z$ on each inner index. While the $Z \otimes Z$ operators in the middle of the loop keep the tensor product state invariant, the two on the two sides of the $y$ direction twist line result in a -1 phase factor due to the presence of the $X \otimes X \alpha$ operator in the $y$ direction. Therefore, for the nontrivial SPT state, the composition of twist lines in the $x$ direction is projective (with a -1 phase factor) in the presence of a twist line in the $y$ direction.

We can interpret this \pm 1 phase factor as related to the $Z_{2}^{2}$ symmetry transformation on each $Z_{2}$ symmetry defect locally that exists at the end of $Z_{2}$ twist lines. For the $Z_{2}$ SPT states, it was shown in Ref. [4] that, by introducing $Z_{2}$ gauge fields and minimally coupling to the original Hamiltonian, the states can be gauged into the toric code and the double semion topological orders, respectively. In particular, the $Z_{2}$ symmetry defects in the bulk are promoted into deconfined excitations by the gauging procedure and they have bosonic and semionic exchange statistics in the trivial and nontrivial SPT state, respectively. Equivalently, we can say that each $Z_{2}$ symmetry defect carries 0 or $1 / 2 Z_{2}$ charge in these two phases. That is, each $Z_{2}$ symmetry defect gets a 1 or $i$ phase factor under the local action of $Z_{2}$ symmetry. If we apply the $Z_{2}$ symmetry locally twice on one $Z_{2}$ symmetry defect, we expect to get a phase factor of 1 and -1 , respectively, which is exactly what we obtained from the twist line composition process discussed above. That is, the projective phase factor coming from the composition of two $Z_{2}$ twist lines in the $x$ direction in the presence of a $Z_{2}$ twist line in the $y$ direction can be interpreted as the $Z_{2}^{2}$ transformation on each $Z_{2}$ symmetry defect locally.

To understand this connection, we can imagine cutting the system open along a nontrivial loop in the $x$ direction and turning the torus into a cylinder, as shown in Fig. 9.

Because of the existence of the $Z_{2}$ twist line in the $y$ direction, $Z_{2}$ symmetry defects are present at the top and bottom end of the cylinder. Now, inserting two $Z_{2}$ twist 


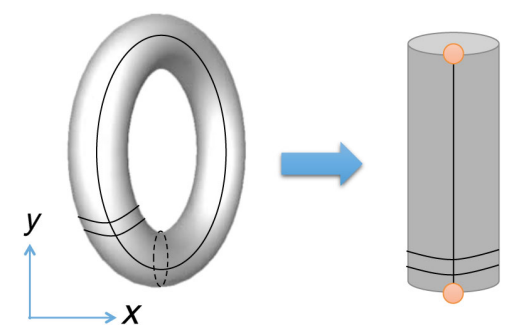

FIG. 9. Cutting the system on a torus open along a nontrivial loop in the $x$ direction (dotted circle) and turning the torus into a cylinder. The $Z_{2}$ twist line in the $y$ direction creates two $Z_{2}$ symmetry defects (orange dots) and the two $Z_{2}$ twist lines in the $x$ direction at the bottom of the cylinder measure the $Z_{2}^{2}$ quantum number of one of the $Z_{2}$ symmetry defects.

lines in the $x$ direction at the bottom end of the cylinder, as show in Fig. 8, is effectively measuring the $Z_{2}^{2}$ quantum number of one $Z_{2}$ symmetry defect. Note that if we actually apply $Z_{2}$ symmetry $\prod \sigma_{x}$ twice in a region near the bottom end, we will not be able to see the nontrivial phase factor because $\left(\prod \sigma_{x}\right)^{2}$ is always equal to 1 . Similar situations happen with time-reversal symmetry in the examples we discuss in later sections.

Similar to the 1D case, this result applies not only to the fixed-point tensors shown in this section but also to those away from the fixed point. The topological properties of the inner symmetry operators remain the same when the tensors are perturbed away from the fixed-point form.

Finally, we comment that there are two differences between the 1D time-reversal SPT example and the 2D $Z_{2}$ SPT example: (1) in composing time-reversal twists, we need to take complex conjugation on one of the twists, while composing unitary $Z_{2}$ twists does not involve such a step, and (2) to see the nontrivial phase factor, for the 1D SPT state we are simply composing two twists, while for the $2 \mathrm{D}$ case we are composing twists in the presence of another twist in the orthogonal direction and thus investigating the relation between them. In our study of 2D phases with time-reversal symmetry, these two features need to be combined.

\section{B. Local action and twists of time reversal in 2D}

Now, we are ready to generalize this procedure to timereversal symmetry in $2 \mathrm{D}$. In $2 \mathrm{D}$, there is only a trivial timereversal symmetric SPT phase $[5,23,24]$ and we use it as an example to illustrate the basic ideas. In Secs. III C and III D, we discuss the more interesting cases of nontrivial SPT phases with $Z_{2} \times Z_{2}^{T}$ symmetry and $Z_{2}$ gauge theories with time-reversal symmetry.

Consider a 2D square lattice with four spin-1/2's per site, and the four spin-1/2's at the corners of each plaquette form an entangled state $|0000\rangle+|1111\rangle$. The total wave function is, hence,

$$
|\psi\rangle=\prod_{\square}(|0000\rangle+|1111\rangle),
$$

where the product is over all plaquettes $\square$. The Hamiltonian of the system can be chosen as

$$
H_{T}=-\sum_{p} \tilde{\sigma}_{x}^{p}
$$

where $\tilde{\sigma}_{x}$ maps between the plaquette spins $|\tilde{0}\rangle=|0000\rangle$ and $|\tilde{1}\rangle=|1111\rangle$.

Time-reversal symmetry acts by first taking complex conjugation in the $|0\rangle,|1\rangle$ basis, and then applying $\sigma_{x}$ to each spin. The state is obviously short-range entangled and time-reversal invariant. We can think of the four spins around a plaquette as a time-reversal domain. The wave function is, hence, an equal weight superposition of all domain configurations. Of course, there are simpler states with the same SPT order, but here we use this form of short-range entangled wave function because this is the standard form of SPT wave function and all SPT states can be written in a similar way. [The $Z_{2}$ SPT state in Eqs. (20) and (22) can also be put into this form with a local basis transformation.]

To define local time-reversal symmetry action, we need the tensor product representation of the state, which gives us a way to divide the coefficient of the wave function into local pieces. The tensors at each site can be chosen as shown in Fig. 10.

With the tensor product representation, we can now define local action of time-reversal symmetry as (1) taking complex conjugation of the tensors (2) acting $\sigma_{x} K$ on the physical basis. The induced inner symmetry operators on the tensors are $X \otimes X$ on each inner index, as shown in Fig. 11. From this relation, we can see that the state is invariant under global time-reversal symmetry action,

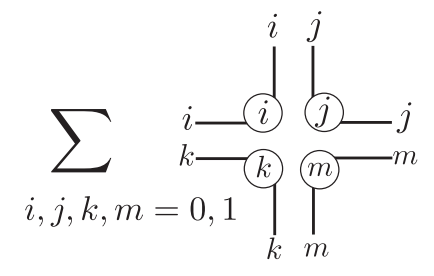

FIG. 10. Tensor representing 2D trivial SPT state with timereversal symmetry. The labels in circles are physical indices and the labels at the end of the links are inner indices.

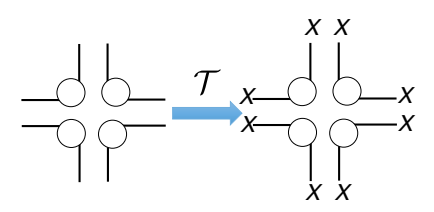

FIG. 11. Acting time reversal on one site induces "inner symmetry operators" $X \otimes X$ on the inner indices. 
because the inner symmetry operators cancel with each other if time reversal is applied globally. Moreover, acting time reversal locally in a subregion changes only the tensors along the border.

Knowing how time reversal acts locally on the state, we can insert twist lines and couple the state to time-reversal fluxes through the nontrivial loops in the system. Similar to the $Z_{2}$ case discussed previously, we expect that

(1) Away from the twist line, the states with and without time-reversal flux should look the same.

(2) Near the twist line, the state with time-reversal flux should look like the state without flux but with timereversal symmetry acting locally in region $R$, where $R$ is a large region with the twist line as part of the border.

For the time-reversal symmetric state discussed above, this can be realized by inserting inner symmetry operators $X \otimes X$ into the inner indices along a nontrivial loop (in the $x$ or $y$ direction). We expect the projective composition rule of time-reversal twist lines to reflect the universal topological properties of the state. Note that because we are considering time-reversal twists here, when composing two inner symmetry operators, we should take complex conjugation of the first one. It is easy to see that two copies of the inner symmetry operators $X \otimes X$ naturally compose into identity; therefore, time-reversal twist lines in this state do not have nontrivial projective composition, as we would expect for a state with trivial SPT order.

Here, we make a comment about how the gauge choice for the tensor product representation affects the result. For unitary symmetries discussed in the previous section, changing the gauge of the tensors does not affect the projective composition rule at all. Suppose that we change the gauge of the tensors by an invertible matrix $N$. The inner symmetry operators all change by conjugation with $N$ and $N^{-1}$ and their composition and commutation relations remain the same.

For the time-reversal symmetry discussed in this section, changing the gauge of the tensor leaves the result almost invariant, except for one subtlety: there are certain gauge choices of the tensors such that local time-reversal symmetry action results in a null state. Suppose that we change the gauge of the upper index of the tensor in Fig. 10 by $e^{i(\pi / 4) X} \otimes I$. Then, applying time-reversal symmetry locally on one site leads to inner symmetry operators $i I \otimes X$ on the upper index and $X \otimes X$ on the other indices. When we try to connect the tensor on different sites and find the total wave function after local time-reversal symmetry action, we find that the resulting state has zero amplitude. Therefore, we need to exclude these possibilities and require that local time-reversal symmetry action always results in a nonzero state.

As long as this nonzero condition is satisfied, gauge change of the tensors does not affect the composition of time-reversal twist lines. For the time-reversal symmetric trivial SPT state discussed above, changing the gauge by an invertible matrix $N$ changes the inner symmetry operators to $N^{*}(X \otimes X) N^{-1}$. Two such operators composed together is still the identity

$$
\left(N^{*}(X \otimes X) N^{-1}\right)^{*} N^{*}(X \otimes X) N^{-1}=I .
$$

Therefore, our discussion is independent of the gauge choice of the tensor product representation (as along as local time-reversal symmetry action results in a nonzero state).

Note that the example wave function we use in this section is a real wave function, for which time reversal is very similar to a unitary $Z_{2}$ symmetry. However, the procedure illustrated by this example applies to complex wave function or complex tensor representations as well. For example, we can choose the wave function to be a product of $i|0000\rangle-$ $i|1111\rangle$ or we can use a complex tensor representation of the original state by adding gauge transformations $S=\operatorname{diag}(1, i)$ and $S^{-1}=\operatorname{diag}(1,-i)$ in pairs to the inner indices. This will not change the projective fusion rule of the time-reversal twist lines and hence, correspondingly, our conclusion about the SPT order.

\section{Example: 2D SPT with $Z_{2} \times Z_{2}^{T}$ symmetry}

Now we study a more interesting example: a 2D SPT state with $Z_{2} \times Z_{2}^{T}$ (time-reversal) symmetry. The $2 \mathrm{D}$ SPT phases with $Z_{2} \times Z_{2}^{T}$ symmetry have a $\mathbb{Z}_{2} \times \mathbb{Z}_{2}$ classification $[5,23,24]$. The trivial and nontrivial SPT order with pure $Z_{2}$ symmetry accounts for the first $\mathbb{Z}_{2}$ in the classification. The second $\mathbb{Z}_{2}$ in the classification corresponds to the $Z_{2}$ symmetry defects in the bulk of the state transforming as $\mathcal{T}^{2}= \pm 1$ under time reversal [29]. (This kind of "local Kramers degeneracy" has also been studied in Ref. [14].) To distinguish states with trivial and nontrivial SPT order under the $Z_{2}$ part of the symmetry, we can insert $Z_{2}$ twist lines along the nontrivial loops and study their projective composition rules as discussed in Sec. III A. In this section, we show how to determine whether $\mathcal{T}^{2}=1$ or -1 on each $Z_{2}$ symmetry defect from the projective composition rule of the time-reversal twist lines in the presence of a unitary $Z_{2}$ twist line.

Consider a $Z_{2} \times Z_{2}^{T}$ SPT state on the square lattice with two sets of spin- $1 / 2$ degrees of freedom $\sigma$ (solid circles in Fig. 12, which we call the $Z_{2}$ spins) and $\tau$ (dashed circles in Fig. 12, which we call the time-reversal spins). The wave function is a product of two parts, the $Z_{2}$ part $\left|\psi_{Z_{2}}\right\rangle$ and the time-reversal part $\left|\psi_{T}\right\rangle$ :

$$
|\psi\rangle=\left|\psi_{Z_{2}}\right\rangle \otimes\left|\psi_{T}\right\rangle .
$$

The $Z_{2}$ part formed by the $\sigma$ spins takes the same form as discussed in Sec. III A, which can have either trivial or nontrivial $Z_{2}$ SPT order. $Z_{2}$ symmetry acts as $\sigma_{x}$ on each $Z_{2}$ spin and does not affect the time-reversal part of the wave 


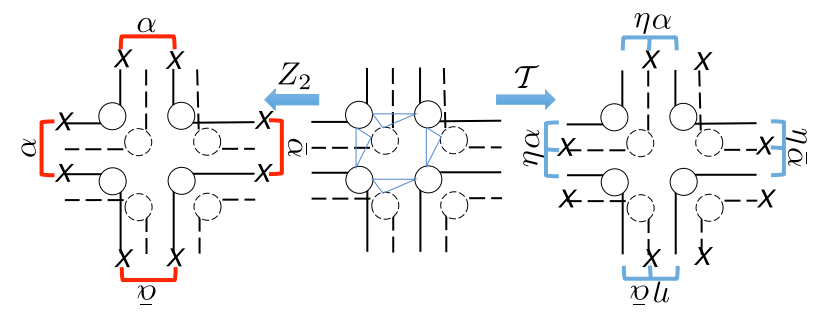

FIG. 12. Local symmetry action on the tensor representing the SPT state with $Z_{2} \times Z_{2}^{T}$ symmetry. The solid $\left(Z_{2}\right)$ part of the tensor follows from that given in Sec. III A and the dashed (timereversal) part of the tensor is the same as that in Fig. 10. Local $Z_{2}$ and time-reversal symmetry induce changes to the tensors as shown on the left- and right-hand side of the figure.

function. The local $Z_{2}$ symmetry action, the $Z_{2}$ symmetry twist line, and their projective composition rules follow directly from Sec. III A, for both the trivial and nontrivial $Z_{2}$ SPT order. The time-reversal part $\left|\psi_{T}\right\rangle$ of the wave function is formed by the $\tau$ spins and is an equal weight superposition of time-reversal domain configurations, as discussed in Sec. III B. The tensor product representation of the time-reversal part is hence the same as that given in Fig. 10. The tensor product representation of the total wave function is a product of the $Z_{2}$ part and the time-reversal part. While the $Z_{2}$ part and the time-reversal part are decoupled in the wave function, they can be intertwined in the definition of time-reversal symmetry action, hence giving rise to possibly nontrivial transformation of $Z_{2}$ symmetry defects under time reversal. We see how this works in detail below.

The SPT order related to the second $\mathbb{Z}_{2}$ in the classification ( $\mathcal{T}^{2}= \pm 1$ on $Z_{2}$ symmetry defects) is encoded in the way time-reversal symmetry acts on the wave function. In the previous section, time reversal acts simply as complex conjugation and $\tau$ spin flip. But, in general, we can have a more complicated action of time reversal by adding another unitary to it as long as applying time reversal twice gives identity. In the following example, the global time-reversal symmetry can act on $\sigma$ and $\tau$ spins together. It is composed of three parts: (1) taking complex conjugation in the $|0\rangle,|1\rangle$ basis of $\sigma$ and $\tau$ spins for the whole wave function, (2) applying $\tau_{x}$ to all $\tau$ spins, and (3) applying phase factors $\eta$ in the $|0\rangle,|1\rangle$ basis to the three spins connected by each triangle in Fig. 12. $\eta$ involves two $\sigma$ spins and one $\tau$ spin and has two possibilities:

$\eta=1$ for all states or

$\eta=-1$ on $|110\rangle$ and $|011\rangle, \quad \eta=1$ otherwise.

The ordering of spins in the definition of $\eta$ is $\sigma \tau \sigma . \eta$ is symmetric with respect to the exchange of the two $\sigma$ 's. If $\eta=1$ for all states, then the $Z_{2}$ part and the time-reversal part of the wave function are decoupled in the time-reversal symmetry action. Therefore, the $Z_{2}$ symmetry defect has to transform trivially as $\mathcal{T}^{2}=1$ under time reversal and, hence, the state has trivial SPT order in the second $\mathbb{Z}_{2}$ classification. With the nontrivial $\eta$, the $Z_{2}$ symmetry defect transforms as $\mathcal{T}^{2}=-1$ and the state has nontrivial SPT order in the second $\mathbb{Z}_{2}$ classification.

First, let us write down a Hamiltonian for the system that respects both $Z_{2}$ and time-reversal symmetry. When $\eta$ is trivial, i.e., when the $Z_{2}$ part and the time-reversal part completely decouples, the Hamiltonian is simply a sum of the $Z_{2}$ part and the time-reversal part,

$$
H_{Z_{2} Z_{2}^{T}}=H_{0}\left(\text { or } H_{1}\right)+H_{T},
$$

where $H_{0}$ and $H_{1}$ act on the $\sigma$ spins and take the form given in Eqs. (21) and (23). $H_{T}$ acts on the $\tau$ spins and takes the form given in Eq. (29). It is straightforward to check that the Hamiltonian defined in this way is symmetric under both the $Z_{2}$ symmetry and the time-reversal symmetry. When $\eta$ is nontrivial, time-reversal symmetry mixes $\sigma$ and $\tau$ spins and $H_{0}\left(H_{1}\right)$ and $H_{T}$ are no longer time-reversal invariant. To restore time-reversal invariance, we need to "dress" each term in $H_{0}\left(H_{1}\right)$ and $H_{T}$ with projectors $P_{\tilde{\sigma}}$ and $P_{\tilde{\tau}}$, which project onto the two-dimensional subspaces of plaquette spins $\tilde{\sigma}$ and $\tilde{\tau}$. For example, the term in $H_{0}$ becomes

$$
-\left(\prod_{q} P_{\tilde{\sigma}}^{q} P_{\tilde{\tau}}^{q}\right) \tilde{\sigma}_{x}^{p}\left(\prod_{q} P_{\tilde{\sigma}}^{q} P_{\tilde{\tau}}^{q}\right)
$$

where the product is over all neighboring plaquettes $q$ around plaquette $p$. It can be checked that the new term is time-reversal invariant even with nontrivial $\eta$. In particular, the projectors force the $\sigma$ spins in the same plaquette to take the same value and the $\tau$ spins in the same plaquette to take the same value. In this kind of configuration, the phase factors $\eta$ involved in the definition of time-reversal symmetry cancel out in pairs at the two ends of the same link. Therefore, time-reversal symmetry acts effectively only on the $\tau$ spins in this projected subspace and time-reversal invariance is guaranteed. The terms in $H_{1}$ and $H_{T}$ can be modified in a similar way.

To understand how the nontrivial $\eta$ is related to the $\mathcal{T}^{2}=-1$ transformation of the $Z_{2}$ symmetry defects, we can think of the time-reversal $\tau$ spin involved in each triangle as living between the $Z_{2}$ domains formed by the two $\sigma$ spins in the same triangle, as shown in Fig. 12. From the definition of $\eta$, we see that if $\tau$ is on a $Z_{2}$ domain wall, then time reversal acts on it as $i \tau_{y} K$, which squares to -1 . If $\tau$ is not on a $Z_{2}$ domain wall, then time reversal acts on it as $\tau_{x} K$, which squares to 1 . Along the domain wall, the $\mathcal{T}^{2}=-1 \tau$ spins form time-reversal singlets and the total wave function is a superposition of all $Z_{2}$ configurations with domain walls decorated by the time-reversal singlets. When $Z_{2}$ symmetry defects are inserted in the bulk of the system, the $Z_{2}$ domain wall ends, leaving unpaired 
$\mathcal{T}^{2}=-1 \tau$ spins at each of the defect point. Therefore, with nontrivial $\eta$, each $Z_{2}$ symmetry defect in the bulk transforms as $\mathcal{T}^{2}=-1$ under time-reversal symmetry.

This feature can be identified from the projective composition rule of the time-reversal twist lines in the state. First, let us find the local time-reversal symmetry action on the representing tensors of the state. The local time-reversal symmetry action on the tensors is given by taking complex conjugation on the tensor and applying $\tau_{x}$, $\eta$, and complex conjugation on the physical basis states. Figure 12 illustrates the inner symmetry operators induced by the local time-reversal symmetry action on the tensor, which reads

$$
M_{\mathcal{T}}=\left(X^{\tau} \otimes X^{\tau}\right) \cdot \eta^{\sigma \tau \sigma} \cdot \alpha^{\sigma \sigma}\left(\text { or } \bar{\alpha}^{\sigma \sigma}\right) .
$$

Here, the $\sigma$ and $\tau$ superscripts label the inner indices in the $Z_{2}$ and time-reversal part of the tensor. The $\alpha^{\sigma \sigma}$ (or $\bar{\alpha}^{\sigma \sigma}$ ) part comes from taking complex conjugation of the $Z_{2}$ part of the tensor, the $X^{\tau} \otimes X^{\tau}$ part comes from the application of $\tau_{x}$, and the $\eta^{\sigma \tau \sigma}$ part comes from the application of the $\eta$ phase factor to the $\sigma$ and $\tau$ spins, as given in Eq. (32).

On the left-hand side of the figure, we also illustrate the inner symmetry operator induced by local $Z_{2}$ symmetry action, which is

$$
M_{Z_{2}}=\left(X^{\sigma} \otimes X^{\sigma}\right) \cdot \alpha^{\sigma \sigma}\left(\text { or } \bar{\alpha}^{\sigma \sigma}\right),
$$

as discussed in Sec. III A.

Suppose that we compose two time-reversal twist lines along the $x$ direction. The composition of two time-reversal inner symmetry operators gives

$$
\begin{aligned}
M_{\mathcal{T}} M_{\mathcal{T}}^{*} & =\left[\left(X^{\tau} \otimes X^{\tau}\right) \cdot \eta^{\sigma \tau \sigma} \cdot \alpha^{\sigma \sigma}\right]\left[\left(X^{\tau} \otimes X^{\tau}\right) \cdot \eta^{\sigma \tau \sigma} \cdot \alpha^{\sigma \sigma}\right]^{*} \\
& =I \quad \text { for trivial } \eta \\
& =Z^{\sigma} \otimes Z^{\sigma} \quad \text { for nontrivial } \eta
\end{aligned}
$$

The result is similar to the composition of two $Z_{2}$ twist lines discussed in Eq. (27). Therefore, if the system has only two time-reversal twist lines in the $x$ direction, then their composition is equivalent to having no twist line.

However, if we are composing two time-reversal twist lines in the $x$ direction in the presence of a $Z_{2}$ symmetry twist line in the $y$ direction, $\mathrm{a}-1$ phase factor arises for nontrivial $\eta$. From Fig. 13 it is easy to see that, when $M_{\mathcal{T}} M_{\mathcal{T}}^{*}=I$ with trivial $\eta$, the composition results in a +1 phase factor, while when $M_{\mathcal{T}} M_{\mathcal{T}}^{*}=Z^{\sigma} \otimes Z^{\sigma}$ with nontrivial $\eta$, the composition results in a -1 phase factor. There is a small complication that we need to explain: the timereversal inner symmetry operator may take a different form $\left(M_{\mathcal{T}}^{\prime}\right)$ at the crossing point with the $Z_{2}$ twist line than everywhere else on the time-reversal twist line $\left(M_{\mathcal{T}}\right)$. This is because with the inserted $Z_{2}$ twist line, local timereversal symmetry action may result in different inner

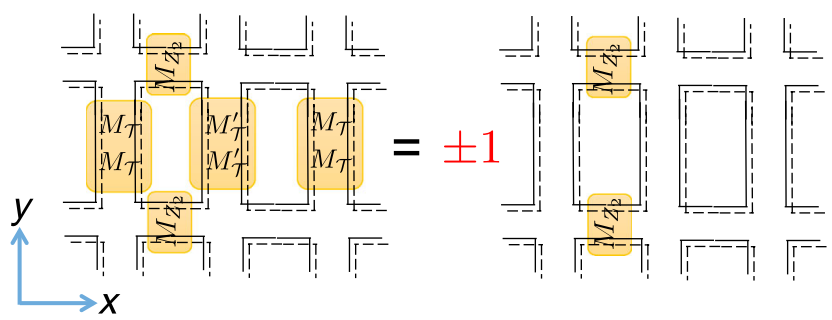

FIG. 13. In the $Z_{2} \times Z_{2}^{T}$ SPT states, composing two timereversal twist lines in the $x$ direction in the presence of a $Z_{2}$ twist line in the $y$ direction is equivalent to a state with only a $y$ direction $Z_{2}$ twist line up to a \pm 1 phase factor, which corresponds to the $\mathcal{T}^{2}= \pm 1$ transformation law on each $Z_{2}$ symmetry defect. The tensor product representation of the state follows from that given in Fig. 12. Physical indices are omitted in the drawing for clarity.

symmetry operators. In particular, as shown in Fig. 14, when $\alpha$ is trivial, $M_{\mathcal{T}}^{\prime}=M_{\mathcal{T}}$; when $\alpha$ is nontrivial, $M_{\mathcal{T}}^{\prime}=M_{\mathcal{T}}\left(Z^{\sigma} \otimes I^{\sigma}\right)$. Explicit calculation shows that our result regarding the composition of time-reversal twist lines is still valid.

In Sec. III A, we interpret the projective phase factor in the composition of two $Z_{2}$ twist lines in the presence of another $Z_{2}$ twist line as the $Z_{2}^{2}$ value of a $Z_{2}$ symmetry defect. Similarly, here we interpret the projective phase factor in the composition of two time-reversal twist lines in the presence of another $Z_{2}$ twist line as the $\mathcal{T}^{2}$ value of a $Z_{2}$ symmetry defect.

Note that the projective phase factor in the composition of two time-reversal twist lines depends on only $\eta$ and not on $\alpha$. This is consistent with the $\mathbb{Z}_{2} \times \mathbb{Z}_{2}$ structure of the $Z_{2} \times Z_{2}^{T}$ SPT classification. $\alpha$ is responsible for the projective phase factor in the composition of two $Z_{2}$ twist lines, which is related to the first $\mathbb{Z}_{2}$ classification while $\eta$ is related to the second.

Finally, we comment that this result is independent of the gauge choice of the representing tensors, as long as local time-reversal action leads to a nonzero state. This is similar to the cases discussed in the previous section.

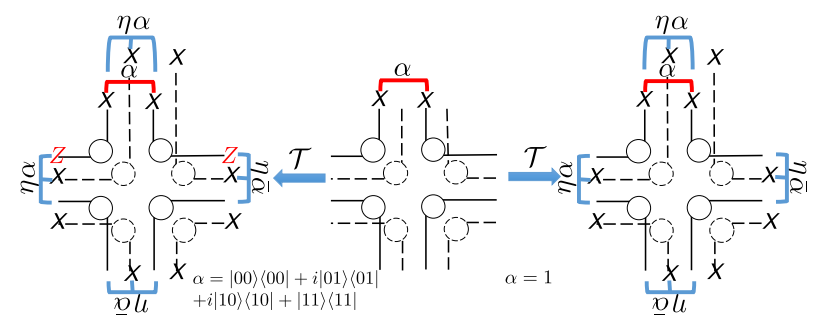

FIG. 14. Local time-reversal action on the tensor representing $Z_{2} \times Z_{2}^{T}$ SPT state with $Z_{2}$ twist line inserted on the top side. When $\alpha$ is trivial (right-hand side), the induced inner symmetry operator remains the same as in Fig. 12; when $\alpha$ is nontrivial (left-hand side), the induced inner symmetry operator changes by $Z^{\sigma} \otimes I^{\sigma}$. 


\section{Example: Toric code and double semion topological order with time-reversal symmetry}

In the previous sections, we study symmetry-protected topological phases with short-range entanglement. In this section, we study an example of a long-range entangled state and demonstrate that the notions of local time-reversal action and time-reversal twist lines still work. In particular, we gauge the $Z_{2}$ symmetry in the examples discussed in the previous section and study $Z_{2}$ gauge theories with timereversal symmetry. These are examples of the so-called symmetry-enriched topological orders $[8,30]$.

In the above discussion, we see that there are four $\left(\mathbb{Z}_{2} \times \mathbb{Z}_{2}\right)$ SPT phases in $2 \mathrm{D}$ with $Z_{2} \times Z_{2}^{T}$ symmetry. Note that even with just $Z_{2}$ symmetry there are two phases. In that case, gauging the $Z_{2}$ symmetry leads to two distinct topological orders: the topological order of the usual $Z_{2}$ gauge theory $\left(Z_{2}\right.$ topological order like in the toric code state [31]) and that of the twisted $Z_{2}$ gauge theory (double semion topological order) [4]. Now, in the presence of timereversal symmetry, the SPT classification implies that there might be an additional twofold distinction. For the usual $Z_{2}$ topological order, this is readily interpreted as arising when each bosonic $Z_{2}$ gauge defect (gauge flux excitation) transforms either linearly with $\mathcal{T}^{2}=1$ or projectively with $\mathcal{T}^{2}=-1$ under time-reversal symmetry. For the twisted $Z_{2}$ gauge theory, such a distinction breaks down because the $Z_{2}$ gauge defects have semionic statistics and are not timereversal invariant. Time reversal acting on the semions turns them into antisemions. Therefore, the $\mathcal{T}^{2}$ value on the gauge defects are no longer well defined and there is only one SET phase with twisted $Z_{2}$ gauge theory and timereversal symmetry [32]. We see below how this distinction between the two types of $Z_{2}$ gauge theories can be extracted by studying the time-reversal symmetry twist lines in the ground state.

A duality transformation from domain degrees of freedom to domain wall degrees of freedom maps a $Z_{2}$ symmetric state to a $Z_{2}$ gauge theory [4]. We still label the $Z_{2}$ spins as $\sigma$ and the time-reversal spins as $\tau$. The tensor product representation of the toric code $Z_{2}$ gauge theory [31] obtained from this mapping is given in Fig. 15(a), while that of the double semion $Z_{2}$ gauge theory is given in Fig. 15(b). The tensor for the toric code state can be obtained from that for the double semion state by dropping all the $i$ and $-i$ phase factors. For simplicity of discussion, we again combine every pair of $A, B$ lattice sites into one $\hat{A}_{\mathrm{B}}^{B} \longrightarrow-$ and map the system to square lattice.

While for the $Z_{2}$ SPT states the representing tensors change by the inner symmetry operators $(X \otimes X) \alpha$ and $(X \otimes X) \bar{\alpha}$ under local $Z_{2}$ action, the tensors for the $Z_{2}$ gauge theories are invariant under such inner symmetry operators, as shown in Fig. 16.

To write down a $Z_{2}$ gauge theory with extra time-reversal structure, we introduce time-reversal spins $\tau$ into the state

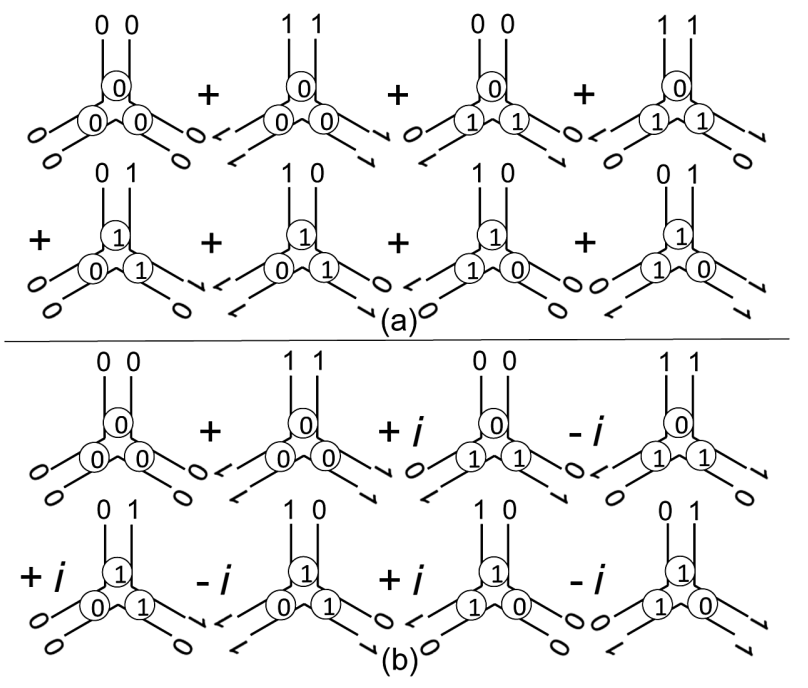

FIG. 15. The tensor product representation of (a) the toric code and (b) the double semion $Z_{2}$ gauge theory. The labels in circles are physical indices and the labels at the end of the links are inner indices.

such that the tensors representing the state are composed of the $Z_{2}$ part given above and the time-reversal part given in Fig. 10. Global time-reversal symmetry action contains three parts: (1) taking complex conjugation in the $|0\rangle,|1\rangle$ basis of the $Z_{2}$ and time-reversal spins, (2) acting $\tau_{x}$ on all the time-reversal spins, and (3) applying phase factors $\eta^{\prime}$ in the $|0\rangle,|1\rangle$ basis to each pair of $Z_{2}$ and time-reversal spin connected by red arrows in Fig. 17. $\eta^{\prime}$ involves one $Z_{2}$ spin and one time-reversal spin and has two possibilities:

$$
\begin{aligned}
& \eta^{\prime}=1 \quad \text { for all states or } \\
& \eta^{\prime}=-1 \text { on }|11\rangle, \quad \eta^{\prime}=1 \text { otherwise. }
\end{aligned}
$$

Note that $\eta^{\prime}$ can be obtained from $\eta$ in Eq. (32) by changing the $Z_{2}$ domain degrees of freedom to the $Z_{2}$ domain wall degrees of freedom.

Time-reversal symmetric Hamiltonians can again be written for these states. The Hamiltonians $H_{Z_{2}^{g} Z_{2}^{T}}$ take a similar form to $H_{Z_{2} Z_{2}^{T}}$, the Hamiltonian of the $Z_{2} \times Z_{2}^{T}$ SPT states, but also have important differences due to the gauging of the $Z_{2}$ symmetry. First, the $Z_{2}$ gauge theory part of the Hamiltonian can be obtained from $H_{0}$ and $H_{1}$, the Hamiltonians for the $Z_{2}$ SPT states, with the duality mapping. It takes a similar form to that given in Ref. [4]:

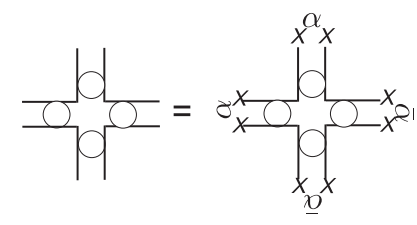

FIG. 16. The tensors representing the $Z_{2}$ gauge theories are invariant under inner symmetry operators $(X \otimes X) \alpha$ and $(X \otimes X) \bar{\alpha}$. 


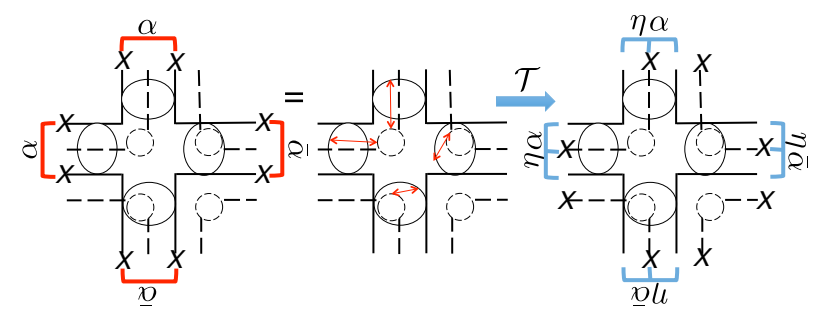

FIG. 17. Local symmetry action on the tensor representing the $Z_{2}$ gauge theory with time-reversal symmetry. The solid $\left(Z_{2}\right)$ part of the tensor follows from that in Fig. 15 and the dashed (time-reversal) part of the tensor is the same as in Fig. 10. Local time-reversal symmetry induces changes to the tensors as shown on the right-hand side of the figure. The left-hand side shows an inner invariance of the tensor related to the $Z_{2}$ gauge symmetry of the state.

$$
H_{0}^{g}=-\sum_{v} \prod_{l \in v} \sigma_{z}^{l}-\sum_{p} \prod_{l \in p} \sigma_{x}^{l}-\sum_{b} \prod_{l \in b} \sigma_{z}^{l}
$$

where the first sum is over all vertices, the second sum over all plaquettes, and the third sum over all bonds (each bond has two spins on it).

$$
\begin{aligned}
H_{1}^{g}= & -\sum_{v} \prod_{l \in v} \sigma_{z}^{l}-\sum_{p} \prod_{l \in p} \sigma_{x}^{l} \prod_{l \in \operatorname{legs} \text { of } p} i^{\left(1-\sigma_{z}^{l}\right) / 2} \\
& -\sum_{b} \prod_{l \in b} \sigma_{z}^{l} .
\end{aligned}
$$

When $\eta$ is trivial, the full Hamiltonian is simply a sum of the $Z_{2}$ gauge theory part and the time-reversal part:

$$
H_{Z_{2}^{g} Z_{2}^{T}}=H_{0}^{g}\left(\text { or } H_{1}^{g}\right)+H_{T}
$$

When $\eta$ is nontrivial, $H_{0}^{g}, H_{1}^{g}$, and $H_{T}$ are no longer timereversal invariant. Instead, we need to dress them up with $P_{\sigma}^{b}$, which projects onto the $|00\rangle$ and $|11\rangle$ subspace of the two $Z_{2} \sigma$ spins on each bond $b$, and $P_{\tilde{\tau}}^{q}$, which projects onto the plaquette spins of $\tau$ 's around a plaquette $q$. For example, the second term in $H_{0}^{g}$ becomes

$$
-\left(\prod_{b} P_{\sigma}^{b} \prod_{q} P_{\tilde{\tau}}^{q}\right) \prod_{l \in p} \sigma_{x}^{l}\left(\prod_{b} P_{\sigma}^{b} \prod_{q} P_{\tilde{\tau}}^{q}\right) ;
$$

the product over $b$ is over all bonds around plaquette $p$, and the product over $q$ is over all neighboring plaquettes around $p$. Other terms in $H_{0}^{g}, H_{1}^{g}$, and $H_{T}$ are modified in a similar way.

Local time-reversal symmetry action then takes complex conjugation of the tensors and applies $\tau_{x}, \eta^{\prime}$, and complex conjugation to the physical degrees of freedom. The transformation of the tensor under local time-reversal action is shown in the right-hand part of Fig. 17. The induced inner symmetry operator is the same as that in the $Z_{2} \times Z_{2}^{T}$ SPT state. The left-hand part of Fig. 17 shows an inner invariance of the tensor, which is related to the $Z_{2}$ gauge symmetry of the state.

Now, we can create time-reversal twist lines by inserting

$$
M_{\mathcal{T}}=\left(X^{\tau} \otimes X^{\tau}\right) \cdot \eta^{\sigma \tau \sigma} \cdot \alpha^{\sigma \sigma}\left(\text { or } \bar{\alpha}^{\sigma \sigma}\right)
$$

into the inner indices along a nontrivial loop. Moreover, we can create $Z_{2}$ gauge twist lines by inserting

$$
M_{Z_{2}}=\left(X^{\sigma} \otimes X^{\sigma}\right) \cdot \alpha^{\sigma \sigma}\left(\text { or } \bar{\alpha}^{\sigma \sigma}\right)
$$

into the inner indices along a nontrivial loop. This way of creating $Z_{2}$ gauge twist lines is equivalent to using the string operators defined in Ref. [33]. Note that, similar to the SPT case discussed in the previous section, when inserting time-reversal symmetry twist lines in the $x$ direction in the presence of a $Z_{2}$ gauge twist line in the $y$ direction, the inner symmetry operator at the crossing point needs to be changed to $M_{\mathcal{T}}^{\prime}$.

As $M_{\mathcal{T}}$ and $M_{Z_{2}}$ are exactly the same as those used in the SPT case, the calculation of the composition between timereversal and $Z_{2}$ gauge twist lines seems to give exactly the same result as shown in Sec. III C. One might want to conclude that there are correspondingly four SET phases. However, there is one major distinction between the SPT and the gauged version. In the gauged version, because the tensor remains invariant under the action of $M_{Z_{2}}$ on the inner indices, the inner symmetry operator induced by local time-reversal symmetry action is not unique. In particular, we can redefine the inner symmetry operator for time reversal as

$$
\tilde{M}_{\mathcal{T}}=M_{\mathcal{T}} M_{Z_{2}}
$$

When calculating the composition of twist lines, we need to do the calculation for both $M_{\mathcal{T}}$ and $\tilde{M}_{\mathcal{T}}$.

When $\alpha$ is trivial, i.e., we have the toric code topological order, $M_{\mathcal{T}}$ and $\tilde{M}_{\mathcal{T}}$ give the same result and we find two types of composition rules for the time-reversal twist lines depending on the choice of $\eta$, similar to what we have seen in the SPT case. We interpret this difference in twist line composition as reflecting the $\mathcal{T}^{2}= \pm 1$ transformation rule on the $Z_{2}$ gauge defects. In the previous discussion, we used a bond dimension- 4 representation of the toric code (as shown in Fig. 15). There is, in fact, a simpler bond dimension-2 representation. We discuss this representation in the Appendix and show that the same construction with twist lines can be used to detect the time-reversal symmetry-enriched topological order.

However, when $\alpha$ is nontrivial, i.e., we have the double semion topological order, $M_{\mathcal{T}}$ and $\tilde{M}_{\mathcal{T}}$ give different results. Consider the composition of two time-reversal 
twist lines in the presence of an orthogonal $Z_{2}$ gauge twist line, similar to the configuration shown in Fig. 13. When $\eta$ is trivial, calculation with $M_{\mathcal{T}}$ yields a phase factor of +1 , while that with $\tilde{M}_{\mathcal{T}}$ gives -1 . On the other hand, when $\eta$ is nontrivial, calculation with $M_{\mathcal{T}}$ gives -1 , while that with $\tilde{M}_{\mathcal{T}}$ gives +1 . Therefore, the distinction between the different choices of $\eta$ disappears. Indeed, there is only one SET phase with double-semion-type topological order and time-reversal symmetry [32]. The $\mathcal{T}^{2}$ value is no longer well defined on the $Z_{2}$ gauge defects in the double semion state as they have semionic statistics and are not invariant under time-reversal symmetry.

\section{CONCLUSION AND OPEN QUESTIONS}

In summary, we propose a way to "gauge" time-reversal symmetry in the tensor network representation of manybody entangled quantum states. First, we define a local action of time-reversal symmetry in the tensor network states. The tensor network representation provides a natural way to divide the global wave function coefficient into local pieces. Based on this division, we can define the local action of complex conjugation, which is the key in defining local actions of antiunitary symmetries. Then we can discuss how to introduce time-reversal twists induced by time-reversal fluxes through the nontrivial loops in the system. Moreover, by composing the time-reversal twists, we can extract topological invariants of the phase from the projective composition rules. In particular, we demonstrate how this works for 1D time-reversal SPT phases, which is a reinterpretation of the procedure used to determine the SPT order from the matrix product state representation of the state. Moreover, we study a 2D timereversal symmetric state with trivial SPT order, 2D SPT states with $Z_{2} \times Z_{2}^{T}$ SPT order, and $2 \mathrm{D} Z_{2}$ gauge theories with time-reversal symmetry. For the $2 \mathrm{D}$ time-reversal symmetric state with trivial SPT order, we find that all the projective composition rules are trivial, as it should be for a trivial SPT state. For the 2D SPT states with $Z_{2} \times Z_{2}^{T}$ SPT order, the projective composition rules of the $Z_{2}$ and time-reversal symmetry twist lines allow us to distinguish all four phases in the $\mathbb{Z}_{2} \times \mathbb{Z}_{2}$ classification. For the $Z_{2}$ gauge theories with time-reversal symmetry, we find three different types of projective composition rules for the $Z_{2}$ gauge twist lines and the time-reversal symmetry twist lines. This allows us to distinguish three SET phases with $Z_{2}$ gauge theory topological order and time-reversal symmetry. Two of them have the toric-code-type topological order with the $Z_{2}$ gauge defect transforming as $\mathcal{T}^{2}=1$ or $\mathcal{T}^{2}=-1$, respectively. The third phase has double semion topological order, and no further distinction can be made based on the time-reversal transformation on the gauge defects [32].

This work just represents our first attempt at gauging time-reversal symmetry, and many questions remain. First of all, our discussion is totally based on quantum states. Is there a way to gauge time-reversal symmetry on the Hamiltonian of the system? For unitary symmetries, we know how to do this. Of course, for time-reversal symmetry we can start from the gauged ground state and construct the corresponding gauged Hamiltonian. However, if we do not know the ground state, do we have a generic way to gauge the Hamiltonian? If we know how to do this, we can gauge time-reversal symmetry not only in gapped systems, but in gapless systems as well.

Second, we ask if there is a dynamical time-reversal gauge theory. In this paper, we are discussing only nondynamical configurations of time-reversal twists. Can we promote it to a dynamical gauge theory by defining a timereversal gauge field and introducing quantum dynamics to it, as can be done for unitary symmetries like $Z_{2}$ ? In the tensor network framework, a representation of the dynamical gauge theory (with unitary gauge groups) can be obtained from that of a symmetric state by imposing the transformation on the tensor resulted from local symmetry actions as a gauge symmetry of the tensor $[34,35]$. Can a similar procedure work for the antiunitary time-reversal symmetry? This is the question we want to study in future work.

Moreover, we want to know what is the general procedure for determining time-reversal-protected and time-reversal-enriched topological orders using timereversal twists. In particular, we want to know how to identify time-reversal-related topological orders in 3D. In $3 \mathrm{D}$, time-reversal symmetry twists take the form of a membrane and time-reversal-related topological orders may be detected by combining time-reversal twist membranes with other gauge or symmetry twist membranes or Wilson loops, as shown in Fig. 18.

In 3D, time-reversal-invariant SPT phases have a $\mathbb{Z}_{2} \times \mathbb{Z}_{2}$ classification. The group cohomology classification gives one $\mathbb{Z}_{2}[23,24]$ and the other $\mathbb{Z}_{2}$ is beyond the group cohomology classification [36,37]. However, as we argue below, a time-reversal twist membrane does not exist for the nontrivial phase in the beyond group cohomology $\mathbb{Z}_{2}$, at least not in the form we would expect.

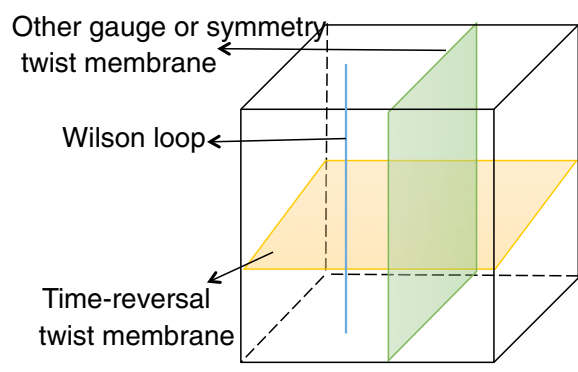

FIG. 18. 3D time-reversal-related topological orders may be detected by combining time-reversal twist membranes with other gauge or symmetry twist membranes or Wilson loops. 
The nontrivial phase in the beyond group cohomology $\mathbb{Z}_{2}$ is special in how time-reversal symmetry acts on the surface of the system. The system has half-quantized surface thermal Hall effect [36]. That is, we can break time-reversal symmetry in opposite ways on the left- and right-hand side of the surface and find a gapless edge state with chiral central charge $c_{-}=8$ between the gapped left and right half. Because time-reversal symmetry on the surface maps the state on the left-hand side to that on the right-hand side, it maps between 2D states with different chirality. This can only be accomplished by a large quantum circuit with circuit depth scaling linearly with the size of the 2D surface. As we can see from all the other examples discussed in this paper, the inner symmetry operators induced by local symmetry actions on the tensors take very similar form to the symmetry transformations on the edge or surface of the state. For all the other examples, the symmetry action on the edge or surface, hence the inner symmetry operators, is composed of a few layers of unitary operators. However, for the beyond group cohomology SPT state, the surface symmetry transformation cannot take this form. Therefore, we expect that the transformation induced by local symmetry action on the tensors cannot be described by simple inner symmetry operators on each inner index, hence complicating the discussion of time-reversal twist membranes in the state.

For the within group cohomology $\mathbb{Z}_{2}$, a time-reversal twist membrane can indeed be found. We leave it to future work to study how to extract topological invariants from it.

Similar ideas may be applied to the study of topological phases with spatial symmetry (reflection, rotation, etc.) as well. In particular, in 1D gapped systems, it is known that the SPT order with spatial reflection symmetry can be extracted from the inner symmetry operators in the matrix product state representation $[17,18]$. Whether similar techniques would allow us to gain more insight into higherdimensional topological phases with spatial symmetry (e.g., topological crystalline insulators [38]) is an interesting question, and we thank the anonymous referee for pointing this out.

Finally, one may ask the question-can gapped quantum ground states be generally represented as tensor network states? This is rigorously known to be true only in 1D [39]. The question of representing chiral 2D states with tensor networks was recently discussed [40,41], although the tensor networks have power-law decaying correlation. Here, we are interested in time-reversal invariant, and hence nonchiral, phases. For example, can topological insulators and superconductors with time-reversal symmetry be represented as short-range correlated tensor network states? If so, will the gauged time-reversal analysis we discuss here give us a deeper understanding of these states? We leave these interesting questions for future work.
Recently, we learned about the work of Kapustin [42], where topological terms for time-reversal gauge field are defined on nonorientable spacetime manifolds.

\section{ACKNOWLEDGMENTS}

X. C. wants to thank Xiao-Gang Wen, Alexei Kitaev, Lukasz Fidkowski, Max Metlitski, and Mike Zaletel for very helpful discussions. X. C. is supported by the Miller Institute for Basic Research in Science at UC Berkeley, the Caltech Institute for Quantum Information and Matter, and the Walter Burke Institute for Theoretical Physics. A. V. is supported by NSF DMR 0645691.

\section{APPENDIX: TORIC CODE WITH BOND DIMENSION-2 REPRESENTATION}

In Sec. III D, we use a bond dimension-4 (double line) representation of the toric code, as shown in Fig. 15. In fact, a simpler bond dimension-2 (single line) representation exists for the toric code. In this Appendix, we discuss how the time-reversal-enriched toric code state can be represented with this single line structure and how a similar construction with twist lines allows us to detect the symmetry-enriched topological order.

The single representation of the toric code is given as in Fig. 19.

Similar to previous discussions, we combine every two sites on the $A, B$ sublattices and map the system to square lattice $\hat{A}_{A}^{B} \longrightarrow+$.

To have time-reversal enrichment, we introduce the $\tau$ spins such that the total tensor representation is a product of the $Z_{2}$ part given above and the time-reversal part given in Fig. 10. Time-reversal symmetry acts on the state as (1) taking complex conjugation in the $|0\rangle,|1\rangle$ basis of the $Z_{2}$ and time-reversal spins, (2) acting $\tau_{x}$ on all the timereversal spins, and (3) applying phase factors $\eta^{\prime}$ in the $|0\rangle$, $|1\rangle$ basis to each pair of $Z_{2}$ and time-reversal spin connected by red arrows in Fig. 20. $\eta^{\prime}$ involves one $Z_{2}$ spin and one time-reversal spin and has two possibilities:

$$
\begin{aligned}
& \eta^{\prime}=1 \quad \text { for all states or } \\
& \eta^{\prime}=-1 \text { on }|11\rangle, \quad \eta^{\prime}=1 \text { otherwise. }
\end{aligned}
$$

On each tensor, time-reversal symmetry acts as shown in Fig. 20, with the induced inner symmetry operator being

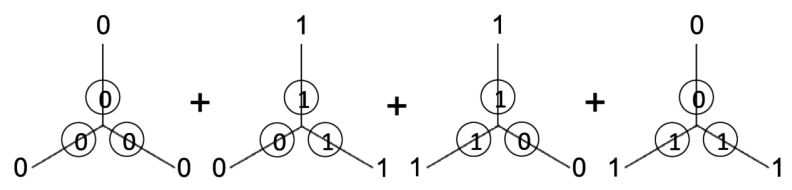

FIG. 19. The single line (bond dimension-2) tensor product representation of the toric code. The labels in circles are physical indices and the labels at the end of the links are inner indices. 


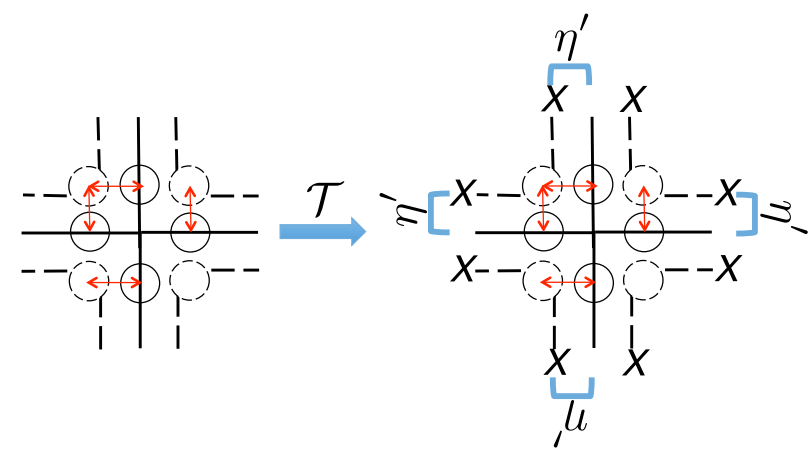

FIG. 20. Time-reversal-enriched single line toric code tensor and its transformation under local time-reversal symmetry action. The solid $\left(Z_{2}\right)$ part of the tensor follows from that in Fig. 19 and the dashed (time-reversal) part of the tensor is the same as in Fig. 10. Time-reversal operation includes phase factors $\eta^{\prime}$ between pairs of $Z_{2}$ and time-reversal spins connected by a red arrow.

$$
M_{T}=\left(X^{\tau} \otimes X^{\tau}\right) \cdot \eta^{\prime \tau \sigma}
$$

Next, we need to find out how the tensor changes when a $Z_{2}$ gauge twist line is inserted. A $Z_{2}$ gauge twist line in the toric code is generated by applying $\sigma_{x}$ to the $Z_{2}$ spins around a loop. If we create a $Z_{2}$ gauge twist line in the $y$ direction around the nontrivial loop, the tensors along the loop change, as shown in Fig. 21, with the induced inner symmetry operator being

$$
M_{Z_{2}}=X^{\sigma}
$$

Note that the single line representation of the toric code is slightly different from the double line representation in this regard. In the double line tensor, the $Z_{2}$ gauge twist line is related to the $Z_{2}$ gauge symmetry of the tensor, as shown in Fig. 17. The single line tensor also has a $Z_{2}$ gauge symmetry, which is applying $Z$ to all four inner indices (solid $Z_{2}$ part) of the tensor. However, this is not related to the $Z_{2}$ gauge twist lines. Instead, we can find the transformation of the tensor
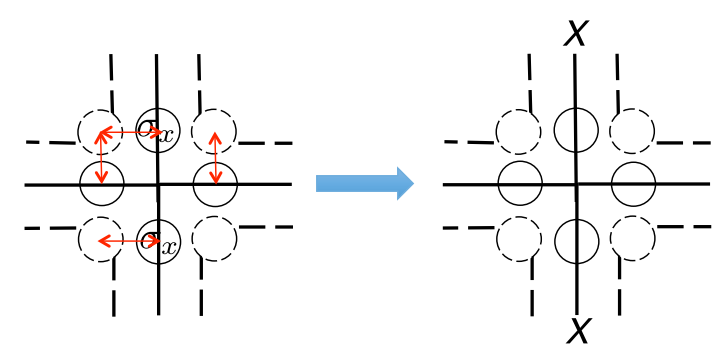

FIG. 21. Time-reversal-enriched single line toric code tensor and its transformation along a $Z_{2}$ gauge twist line in the $y$ direction.

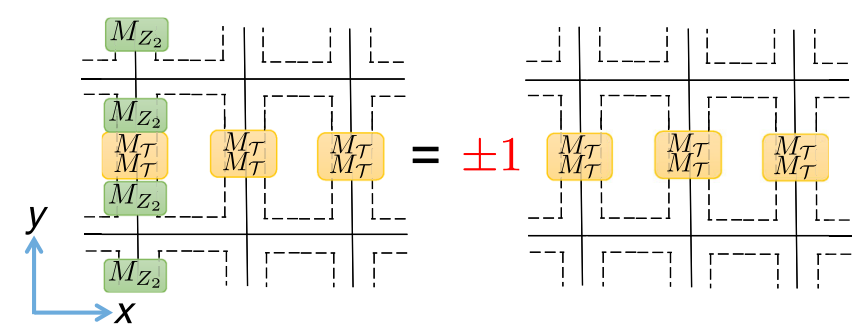

FIG. 22. In the time-reversal-enriched single line toric code tensor product state, inserting a $Z_{2}$ gauge twist line in the $y$ direction in the presence of two time-reversal twist lines in the $x$ direction is equivalent to a state with only the time-reversal twist lines up to a \pm 1 phase factor, which corresponds to the $\mathcal{T}^{2}= \pm 1$ transformation law on each $Z_{2}$ gauge defect. The tensor product representation of the state follows from that given in Fig. 20. Physical indices are omitted in the drawing for clarity.

along the $Z_{2}$ gauge twist line by physically applying the string operator of $\sigma_{x}$ 's and see how the tensor changes. (We can do the same with the double line tensor and we would get the same result.)

Now we can put the $Z_{2}$ gauge twist line and the timereversal symmetry twist lines together and try to detect the symmetry-enriched topological order. As shown in Fig. 22, inserting a $Z_{2}$ gauge twist line in the $y$ direction in the presence of two time-reversal twist lines in the $x$ direction is equivalent to a state with only the time-reversal twist lines up to $\mathrm{a} \pm 1$ phase factor. In particular, the phase factor is +1 with trivial $\eta^{\prime}$ and the phase factor is -1 with nontrivial $\eta^{\prime}$ [see Eq. (A1)]. Therefore, the \pm 1 phase factor indicates different symmetry-enriched topological order, similar to the result obtained in Sec. III D using the double line representation.

We want to make a few comments here. First, the inner symmetry operator for time reversal remains invariant in the presence of an inserted $Z_{2}$ gauge twist line. Therefore, we do not need to modify $M_{T}$ at the intersection with the $Z_{2}$ twist line. Second, $M_{T}$ and $M_{Z_{2}}$ can each be modified by the gauge symmetry operator $Z^{\sigma}$ of the single line tensor, but the result does not change. Therefore, the \pm 1 phase factors are truly invariants of symmetry-enriched topological phases. Third, one may notice that the calculation in Fig. 22 is slightly different from that in Fig. 13. In Fig. 13 the right-hand side contains the $Z_{2}$ gauge twist line in the $y$ direction, while in Fig. 22 the right-hand side contains the time-reversal twist lines in the $x$ direction. To make the calculation process consistent with each other, we can simply take the phase difference between different orders of applying the twist lines. That is, we can apply the $y$ direction $Z_{2}$ gauge twist line first and the $x$ direction time-reversal twist lines second or vice verse. The phase difference of these two processes will be \pm 1 , which gives the topological invariant for both single line and double line representations. 
[1] R. B. Laughlin, Quantized Hall Conductivity in Two Dimensions, Phys. Rev. B 23, 5632 (1981).

[2] N. Read and D. Green, Paired States of Fermions in Two Dimensions with Breaking of Parity and Time-Reversal Symmetries and the Fractional Quantum Hall Effect, Phys. Rev. B 61, 10267 (2000).

[3] C. N. Yang and R. L. Mills, Conservation of Isotopic Spin and Isotopic Gauge Invariance, Phys. Rev. 96, 191 (1954).

[4] M. Levin and Z.-C. Gu, Braiding Statistics Approach to Symmetry-Protected Topological Phases, Phys. Rev. B 86, 115109 (2012).

[5] Y.-M. Lu and A. Vishwanath, Theory and Classification of Interacting Integer Topological Phases in Two Dimensions: A Chern-Simons Approach, Phys. Rev. B 86, 125119 (2012).

[6] Z.-C. Gu and M. Levin, Effect of Interactions on TwoDimensional Fermionic Symmetry-Protected Topological Phases with $Z_{2}$ Symmetry, Phys. Rev. B 89, 201113 (2014).

[7] M. Cheng and Z.-C. Gu, Topological Response Theory of Abelian Symmetry-Protected Topological Phases in Two Dimensions, Phys. Rev. Lett. 112, 141602 (2014).

[8] Y.-M. Lu and A. Vishwanath, Classification and Properties of Symmetry Enriched Topological Phases: A ChernSimons Approach with Applications to $Z_{2}$ Spin Liquids, arXiv:1302.2634.

[9] Z.-X. Liu and X.-G. Wen, Symmetry-Protected Quantum Spin Hall Phases in Two Dimensions, Phys. Rev. Lett. 110, 067205 (2013).

[10] L.-Y. Hung and X.-G. Wen, Quantized Topological Terms in Weak-Coupling Gauge Theories with a Global Symmetry and Their Connection to Symmetry-Enriched Topological Phases, Phys. Rev. B 87, 165107 (2013).

[11] J. I. Cirac and F. Verstraete, Renormalization and Tensor Product States in Spin Chains and Lattices, J. Phys. A 42, 504004 (2009).

[12] G. Vidal, Entanglement Renormalization: An Introduction, arXiv:0912.1651.

[13] In Ref. [14] a different notion of local time reversal symmetry action is defined that applies not to ground states but to fractional excitations in topological phases.

[14] M. Levin and A. Stern, Classification and Analysis of TwoDimensional Abelian Fractional Topological Insulators, Phys. Rev. B 86, 115131 (2012).

[15] As we are interested in gapped quantum phases with local interaction in this paper, we focus on tensor networks with finite bond dimension and finite bond length.

[16] Z.-C. Gu and X.-G. Wen, Tensor-Entanglement-Filtering Renormalization Approach and Symmetry-Protected Topological Order, Phys. Rev. B 80, 155131 (2009).

[17] F. Pollmann, A. M. Turner, E. Berg, and M. Oshikawa, Entanglement Spectrum of a Topological Phase in One Dimension, Phys. Rev. B 81, 064439 (2010).

[18] F. Pollmann, E. Berg, A. M. Turner, and M. Oshikawa, Symmetry Protection of Topological Phases in OneDimensional Quantum Spin Systems, Phys. Rev. B 85, 075125 (2012).

[19] X. Chen, Z.-C. Gu, and X.-G. Wen, Classification of Gapped Symmetric Phases in One-Dimensional Spin Systems, Phys. Rev. B 83, 035107 (2011).
[20] N. Schuch, D. Perez-Garcia, and I. Cirac, Classifying Quantum Phases Using Matrix Product States and Projected Entangled Pair States, Phys. Rev. B 84, 165139 (2011).

[21] S. Bravyi, M. B. Hastings, and S. Michalakis, Topological Quantum Order: Stability under Local Perturbations, J. Math. Phys. (N.Y.) 51, 093512 (2010).

[22] X. Chen, Z.-X. Liu, and X.-G. Wen, Two-Dimensional Symmetry-Protected Topological Orders and Their Protected Gapless Edge Excitations, Phys. Rev. B 84, 235141 (2011).

[23] X. Chen, Z.-C. Gu, Z.-X. Liu, and X.-G. Wen, SymmetryProtected Topological Orders in Interacting Bosonic Systems, Science 338, 1604 (2012).

[24] X. Chen, Z.-C. Gu, Z.-X. Liu, and X.-G. Wen, Symmetry Protected Topological Orders and the Group Cohomology of Their Symmetry Group, Phys. Rev. B 87, 155114 (2013).

[25] Z.-C. Gu, M. Levin, B. Swingle, and X.-G. Wen, TensorProduct Representations for String-Net Condensed States, Phys. Rev. B 79, 085118 (2009).

[26] To see that the tensor given in Fig. 4(b) correctly produces the wave function in Eq. (22), notice that on a honeycomb lattice one makes left and right turns along a loop. To make a full loop, the number of left turns differs from the number of right turns by 6 . The left and right turns are associated with phase factors of $i$ and $-i$, respectively; therefore, each loop obtains a phase factor of -1 .

[27] L.-Y. Hung and X.-G. Wen, Universal Symmetry-Protected Topological Invariants for Symmetry-Protected Topological States, Phys. Rev. B 89, 075121 (2014).

[28] H. Moradi and X.-G. Wen, Universal Wave Function Overlap and Universal Topological Data from Generic Gapped Ground States, Phys. Rev. Lett. 115, 036802 (2015).

[29] X. Chen, Y.-M. Lu, and A. Vishwanath, SymmetryProtected Topological Phases from Decorated Domain Walls, Nat. Commun. 5, 3507 (2014).

[30] A. M. Essin and M. Hermele, Classifying Fractionalization: Symmetry Classification of Gapped $\mathbb{Z}_{2}$ Spin Liquids in Two Dimensions, Phys. Rev. B 87, 104406 (2013).

[31] A. Yu. Kitaev, Fault-Tolerant Quantum Computation by Anyons, Ann. Phys. (Amsterdam) 303, 2 (2003).

[32] F. J. Burnell, X. Chen, A. Kitaev, M. A. Metlitski, and A. Vishwanath, Time Reversal Invariant Gapped Boundaries of the Double Semion State (to be published).

[33] M. A. Levin and X.-G. Wen, String-Net Condensation: A Physical Mechanism for Topological Phases, Phys. Rev. B 71, 045110 (2005).

[34] L. Tagliacozzo, A. Celi, and M. Lewenstein, Tensor Networks for Lattice Gauge Theories with Continuous Groups, Phys. Rev. X 4, 041024 (2014).

[35] J. Haegeman, K. Van Acoleyen, N. Schuch, J. I. Cirac, and F. Verstraete, Gauging Quantum States: From Global to Local Symmetries in Many-Body Systems, Phys. Rev. X 5 , 011024 (2015).

[36] A. Vishwanath and T. Senthil, Physics of Three-Dimensional Bosonic Topological Insulators: 
Surface-Deconfined Criticality and Quantized Magnetoelectric Effect, Phys. Rev. X 3, 011016 (2013).

[37] A. Kitaev (private communication).

[38] L. Fu, Topological Crystalline Insulators, Phys. Rev. Lett. 106, 106802 (2011).

[39] M. B. Hastings, An Area Law for One-Dimensional Quantum Systems, J. Stat. Mech. (2007) P08024.

[40] J. Dubail and N. Read, Tensor Network Trial States for Chiral Topological Phases in Two Dimensions and a
No-Go Theorem in Any Dimension, arXiv:1307.7726 [Phys. Rev. B (to be published)].

[41] T. B. Wahl, H.-H. Tu, N. Schuch, and J. I. Cirac, Projected Entangled-Pair States Can Describe Chiral Topological States, Phys. Rev. Lett. 111, 236805 (2013).

[42] A. Kapustin, Symmetry Protected Topological Phases, Anomalies, and Cobordisms: Beyond Group Cohomology, arXiv:1403.1467. 